\title{
A RIGOROUS APPROACH TO THE FEYNMAN-VERNON INFLUENCE FUNCTIONAL AND ITS APPLICATIONS. I
}

\author{
S. ALBEVERIO ${ }^{1,2), 3}$, L. CATTANEO ${ }^{1)}$, L. DI PERSIO ${ }^{2)}$, S. MAZZUCCHI ${ }^{2,4)}$
}

Keywords: Oscillatory integrals, Feynman path integrals, FeynmanVernon influence functional, Caldeira-Leggett model, open quantum systems.

AMS classification : 28, 35C15, 81C35, 81S40, 81Q05.

\begin{abstract}
A rigorous representation of the Feynman-Vernon influence functional used to describe open quantum systems is given, based on the theory of infinite dimensional oscillatory integrals. An application to the case of the density matrices describing the Caldeira-Leggett model of two quantum systems with a quadratic interaction is treated.
\end{abstract}

\section{INTRODUCTION}

One of the crucial problems of modern physics consists in understanding the behaviour of an open quantum system, i.e. of a quantum system coupled with a second system often called reservoir or enviroment. One is interested in the dynamics of the first system, taking into account the influence of the enviroment on it. A typical example is the study of a quantum particle submitted to the measurement of an observable. In fact, from a quantum mechanical point of view, the interaction with the measuring apparatus cannot be neglected and modifies the dynamics of the particle. On the other hand the evolution of the measuring instrument is not of primary interest.

A particularly intriguing approach to this problem was proposed in 1963 by Feynman and Vernon ( see [FH65, FV63]) within the path integral formulation of quantum mechanics. In 1942 R.P. Feynman [Fey42], following a suggestion by Dirac (see [Dir33, Dir47], proposed an alternative (Lagrangian) formulation of quantum mechanics (published in [Fey48]), that is an heuristic, but very suggestive representation for the 
solution of the Schrödinger equation

$$
\left\{\begin{array}{l}
i \hbar \frac{\partial}{\partial t} \psi=-\frac{\hbar^{2}}{2 M} \Delta \psi+V \psi \\
\psi(0, x)=\psi_{0}(x)
\end{array}\right.
$$

describing the time evolution of the state $\psi$ of a $d$-dimensional quantum particle. The parameter $\hbar$ is the reduced Planck constant, $m>0$ is the mass of the particle and $F=-\nabla V$ is an external force. According to Feynman's proposal the wave function of the system at time $t$ evaluated at the point $x \in \mathbb{R}^{d}$ is heuristically given as an "integral over histories", or as an integral over all possible paths $\gamma$ in the configuration space of the system with finite energy passing in the point $x$ at time $t$ :

$$
\psi(t, x)="\left(\int_{\{\gamma \mid \gamma(t)=x\}} e^{\frac{i}{\hbar} S_{t}^{\circ}(\gamma)} D \gamma\right)^{-1} \int_{\{\gamma \mid \gamma(t)=x\}} e^{\frac{i}{\hbar} S_{t}(\gamma)} \psi_{0}(\gamma(0)) D \gamma "
$$

where $S_{t}(\gamma)$ is the classical action of the system evaluated along the path $\gamma$, i.e. :

$$
\begin{gathered}
S_{t}(\gamma) \equiv S_{t}^{\circ}(\gamma)-\int_{0}^{t} V(\gamma(s)) d s \\
S_{t}^{\circ}(\gamma) \equiv \frac{M}{2} \int_{0}^{t}|\dot{\gamma}(s)|^{2} d s
\end{gathered}
$$

$D \gamma$ is an heuristic Lebesgue "flat" measure on the space of paths and $\left(\int_{\{\gamma \mid \gamma(t)=x\}} e^{\frac{i}{\hbar} S_{t}^{\circ}(\gamma)} D \gamma\right)^{-1}$ is a normalization constant.

Feynman and Vernon (see [FH65, FV63]) generalized this idea to the study of the time evolution of the reduced density operator of a system in interaction with an enviroment. Let denote $\rho_{A}, \rho_{B}$, respectively, the initial density matrices of the system and of the enviroment, $S_{A}, S_{B}$, respectively, the action functionals of the system and of the enviroment and $S_{I}$ the contribution to the total action due to the interaction. Then the kernel of the reduced density operator of the system $\rho_{R}$ (obtained by tracing over the environmental coordinates) is heuristically given by:

$$
\rho_{R}(t, x, y)=" \int_{\substack{\gamma(t)=x \\ \gamma^{\prime}(t)=y}} e^{\frac{i}{\hbar}\left(S_{A}(\gamma)-S_{A}\left(\gamma^{\prime}\right)\right)} F\left(\gamma, \gamma^{\prime}\right) \rho_{A}\left(\gamma(0), \gamma^{\prime}(0)\right) D \gamma D \gamma^{\prime} \quad "
$$

where $F$ is the formal influence functional (IF):

$$
\begin{aligned}
F\left(\gamma, \gamma^{\prime}\right)=" \int_{\substack{\Gamma(t)=Q \\
\Gamma^{\prime}(t)=Q}} e^{\frac{i}{\hbar}\left(S_{B}(\Gamma)-S_{B}\left(\Gamma^{\prime}\right)\right)} e^{\frac{i}{\hbar}\left(S_{I}(\Gamma, \gamma)-S_{I}\left(\Gamma^{\prime}, \gamma^{\prime}\right)\right)} \times \\
\times \rho_{B}\left(\Gamma(0), \Gamma^{\prime}(0)\right) D \Gamma D \Gamma^{\prime} d Q "
\end{aligned}
$$


The number of spin-offs originated by the seminal work [FV63] is so large that it is nearly impossible to give here a complete list, and we limit ourselves to shortly mention some of them.

Probably the most influential contributions can be found in [CL83a, CL83b] where Caldeira and Leggett applied the heuristic IF method in order to study the quantum Brownian motion (QBM) (i.e. the analogue of Brownian motion of a quantum particle) and the tunneling phenomenon in dissipative systems. Latter papers triggered a chain-reaction which is actually far from its end. In [Leg84] (see also [CL81, Cal83]) Leggett determined the imaginary-time functional which supplies the tunnelling rate form of a metastable state at zero temperature, in a formal WKB limit, in presence of an arbitrary linear dissipation mechanism. In [CL85] an explicit calculation of the time-dependent density matrix is given describing the damping on quantum interference between two gaussian wave packets in a harmonic potential and the obtained results are in agreement with the quantum theory of measurement, see e.g. [Zur82].

In [HA85] the decoupled particle-bath initial condition previously used, was compared with the initial off-diagonal coherence of the reduced density matrix, constituting the thermal initial condition.

A wide-range use of the IF approach was given in [ $\left.\mathrm{LCD}^{+} 87\right]$ where the authors mixed their previous experiences giving a deep view to the dynamics of a two-state system coupled to a dissipative environment.

In [CH87] an application of the IF formalism was given in order to study the reduced density operator of a particle coupled with a fermionic environment. Similar applications may be found in [Sch82, Gui84, Che87, Zwe87, BSZ92], where the fluctuations in the motion of a heavy particle interacting with a free fermion gas are studied providing various type of classical and semiclassical expansion either with and without weak-potential or linear response assumptions.

Chen's approach was extended in the case of a boson bath in [CLL89].

The heuristic IF approach was generalized in [SC87, SC90] to a nonfactorizable initial system-plus-reservoir density operator without specific symmetry assumptions.

Since heterogeneous problems related to macroscopic effects in quantum system require extensions to the QBM theory, following [CL83a] various attempts to derive a master-equation (ME) were made in order to include general initial conditions and nonlinear couplings. The ME for linear coupling and ohmic environment at high temperature found in [CL83a] was first extended to arbitrary temperature in [UZ89] and afterwards obtained for more general environments and nonlocal 
couplings, which produce colored noise and nonlocal dissipation, see [GSI88, HPZ92, Bru93, HPZ93, BG03] and references therein.

A complementary use of the IF approach to the description of Markovian open quantum systems can be found in [Str97] where the IF method is used in order to develop the ME of general Lindblad positivesemigroup (see [Lin76]) and the propagator in a formal stationary phase approximation is calculated.

Actually the derivation of the ME for the reduced dynamics of quantum system have gained a lot of contributions by the use of mathematical respectively physical path integrals (PI) techniques (see e.g. [Exn85, JL02] respectively [Wei99, BP02, Kle04, GZ04] and references therein).

The IF formalism was also used in parametric random matrices approach to the problem of dissipation in many-body systems, see e.g. [BDK95, BDK96, BDK97, BDK98] and reference therein, where the derived form of the IF differs from the one in [CL83a] and recovers the latter as the first term of its formal Taylor expansion.

The emerging theory of Quantum Computation is another field of application of the IF method since the implementation of real quantum processors is often hampered by the quantum decoherence phenomenon, see e.g. [Deu89, Unr95, BDE95, DS98, PZ99, GJZ+ 03, SH04] and references therein.

Despite the broad range of its applications, a rigorous mathematical construction of the IF is still missing.

Our aim is to fill this gap following the ideas introduced in [AHK76, AHK77] in connection with the rigorous mathematical definition of Feynman path integrals (2) and in order to realize formulae (5) and (6) as well defined infinite dimensional oscillatory integrals on a suitable Hilbert space.

Before we go over to a short description of our present work we would like to outline that there are rigorous works on models of particles in interaction with heat bath not based on the IF approach, e.g. see [Dav73, CEFM00] and references therein.

In Section 2 we recall some known results, extend the definition of infinite dimensional oscillatory integrals and prove some important properties (see [AGM03, AGM04, AM05b, AM05a, AM04a, AM04b, AM] and references therein). In Section 3 the new functional integral is used in the study of the time evolution of two linearly interacting quantum 
systems. A mathematical formalization of the Feynman-Vernon's theory of the IF is given in Section 4 The main results of the paper are Theorems (6) and (7) where a conseguence of the appendix A is used in order to prove the integrability of certain function. The last section is devoted to the study of the Caldeira-Leggett model (see [CL83a]) in the case of a finite dimensional heat bath.

In the second part of this work we shall use our representation of the IF to study rigorously the limit of an infinite dimensional heat bath and of various physically relevant limits, partly discussed in above physical literature's references.

\section{INFINITE DIMENSIONAL OSCILLATORY INTEGRALS}

In this section we give the definitions of infinite dimensional oscillatory integrals and prove some important properties which will be used in the application to the study of the time evolution of a quantum system.

In the following we shall denote by $\mathcal{H}$ a (finite or infinite dimensional) real separable Hilbert space, whose elements will be denoted by $x, y \in$ $\mathcal{H}$ and the scalar product with $\langle x, y\rangle . f: \mathcal{H} \rightarrow \mathbb{C}$ will be a function on $\mathcal{H}$ and $L: D(L) \subseteq \mathcal{H} \rightarrow \mathcal{H}$ an invertible, densely defined and selfadjoint operator.

Let us denote by $\mathcal{M}(\mathcal{H})$ the Banach space of the complex bounded variation measures on $\mathcal{H}$, endowed with the total variation norm, that is:

$$
\mu \in \mathcal{M}(\mathcal{H}), \quad\|\mu\|=\sup \sum_{i}\left|\mu\left(E_{i}\right)\right|
$$

where the supremum is taken over all sequences $\left\{E_{i}\right\}$ of pairwise disjoint Borel subsets of $\mathcal{H}$, such that $\cup_{i} E_{i}=\mathcal{H} . \mathcal{M}(\mathcal{H})$ is a Banach algebra, where the product of two measures $\mu * \nu$ is by definition their convolution:

$$
\mu * \nu(E)=\int_{\mathcal{H}} \mu(E-x) \nu(d x), \quad \mu, \nu \in \mathcal{M}(\mathcal{H})
$$

and the unit element is the vector $\delta_{0}$.

Let $\mathcal{F}(\mathcal{H})$ be the space of complex functions on $\mathcal{H}$ which are Fourier transforms of measures belonging to $\mathcal{M}(\mathcal{H})$, that is:

$$
f: \mathcal{H} \rightarrow \mathbb{C} \quad f(x)=\int_{\mathcal{H}} e^{i\langle x, \beta\rangle} \mu_{f}(d \beta) \equiv \hat{\mu}_{f}(x) .
$$

$\mathcal{F}(\mathcal{H})$ is a Banach algebra of functions, where the product is the pointwise one; the unit element is the function 1, i.e. $1(x)=1 \forall x \in \mathcal{H}$ and the norm is given by $\|f\|=\left\|\mu_{f}\right\|$. 
The study of oscillatory integrals on $\mathbb{R}^{n}$ with quadratic phase functions, i.e. the "Fresnel integrals",

$$
\int e^{\frac{i}{2 \hbar}\langle x, x\rangle} f(x) d x, \quad \hbar>0
$$

is a largely developed topic, and has strong connections with several problems in mathematics, e.g. in the theory of Fourier integral operators, and physics, e.g. in optics. Following Hörmander, the integral in (7) can be defined even if $f(\mathcal{H})$ is not summable by exploiting the cancellations due to the oscillatory behavior of the integrand, by means of a limiting procedure. More precisely the Fresnel integrals can be defined as the limit of a sequence of regularized, hence absolutely convergent, Lebesgue integrals.

Definition 1. A function $f: \mathbb{R}^{n} \rightarrow \mathbb{C}$ is Fresnel integrable if and only if for each $\phi \in \mathcal{S}\left(\mathbb{R}^{n}\right)$ such that $\phi(0)=1$ the limit

$$
\lim _{\epsilon \rightarrow 0}(2 \pi i \hbar)^{-n / 2} \int e^{\frac{i}{2 \hbar}\langle x, x\rangle} f(x) \phi(\epsilon x) d x
$$

exists and is independent of $\phi$. In this case the limit is called the Fresnel integral of $f$ and denoted by

$$
\widetilde{\int} e^{\frac{i}{2 \hbar}\langle x, x\rangle} f(x) d x
$$

In [ET84] this definition was generalized to the case $\mathbb{R}^{n}$ is replaced by an infinite dimensional real separable Hilbert space $\mathcal{H}$. In fact an infinite dimensional Fresnel integral can be defined as the limit of a sequence of finite dimensional approximations:

Definition 2. Let $(\mathcal{H},\langle\rangle$,$) be a real separable (infinite dimensional)$ Hilbert space. A function $f: \mathcal{H} \rightarrow \mathbb{C}$ is Fresnel integrable if and only if for any sequence $P_{n}$ of projectors onto $n$-dimensional subspaces of $\mathcal{H}$, such that $P_{n} \leq P_{n+1}$ and $P_{n} \rightarrow 1$ strongly as $n \rightarrow \infty$ (1 being the identity operator in $\mathcal{H})$, the finite dimensional approximations

$$
(2 \pi i \hbar)^{-n / 2} \int_{P_{n} \mathcal{H}} e^{\frac{i}{2 \hbar}\left\langle P_{n} x, P_{n} x\right\rangle} f\left(P_{n} x\right) d\left(P_{n} x\right),
$$

are well defined (in the sense of definition 1) and the limit

$$
\lim _{n \rightarrow \infty}(2 \pi i \hbar)^{-n / 2} \int_{P_{n} \mathcal{H}} e^{\frac{i}{2 \hbar}\left\langle P_{n} x, P_{n} x\right\rangle} f\left(P_{n} x\right) d\left(P_{n} x\right)
$$

exists and is independent of the sequence $\left\{P_{n}\right\}$.

In this case the limit is called the Fresnel integral of $f$ and is denoted 
by:

$$
\widetilde{\int} e^{\frac{i}{2 \hbar}\langle x, x\rangle} f(x) d x
$$

A complete " direct description" of the largest class of Fresnel integrable functions is still an open problem, even in finite dimension. However it is possible to find some interesting subsets of it, as the following result shows.

Theorem 1. Let $L: \mathcal{H} \rightarrow \mathcal{H}$ be a self adjoint trace-class operator, such that $(I-L)$ is invertible. Let $y \in \mathcal{H}$ and let $f: \mathcal{H} \rightarrow \mathbb{C}$ be the Fourier transform of a complex bounded variation measure $\mu_{f}$ on $\mathcal{H}$. Then the function $e^{-\frac{i}{2 \hbar}\langle x, L x\rangle} e^{i\langle x, y\rangle} f(x)$ is Fresnel integrable and the corresponding Fresnel integral can be explicitly computed in terms of a well defined absolutely convergent integral with respect to a $\sigma$-additive measure, by means of the following Parseval-type equality:

$$
\begin{aligned}
& \widetilde{\int} e^{\frac{i}{2 \hbar}\langle x, x\rangle} e^{-\frac{i}{2 \hbar}\langle x, L x\rangle} e^{i\langle x, y\rangle} f(x) d x= \\
& =(\operatorname{det}(I-L))^{-1 / 2} \int_{\mathcal{H}} e^{-\frac{i \hbar}{2}\left\langle\alpha+y,(I-L)^{-1}(\alpha+y)\right\rangle} \mu_{f}(d \alpha)
\end{aligned}
$$

where $\operatorname{det}(I-L)=|\operatorname{det}(I-L)| e^{-\pi i \operatorname{Ind}(I-L)}$ is the Fredholm determinant of the operator $(I-L),|\operatorname{det}(I-L)|$ its absolute value and $\operatorname{Ind}((I-L))$ is the number of negative eigenvalues of the operator $(I-L)$, counted with their multiplicity.

Proof: The result follows directly by theorem 2.1 in [AB93], see also [ET84], which states that for $g \in \mathcal{F}(\mathcal{H})$

$$
\widetilde{\int} e^{\frac{i}{2 \hbar}\langle x, x\rangle} e^{-\frac{i}{2 \hbar}\langle x, L x\rangle} g(x) d x=\frac{1}{\sqrt{\operatorname{det}(I-L)}} \int_{\mathcal{H}} e^{-\frac{i \hbar}{2}\left\langle\alpha,(I-L)^{-1}(\alpha)\right\rangle} \mu_{g}(d \alpha)
$$

By taking $\mu_{g}:=\delta_{y} * \mu_{f}$ the conclusion follows.

By expression (11) the following result follows easily:

Corollary 1. Under the assumptions of theorem 1, the functional

$$
f \in \mathcal{F}(\mathcal{H}) \mapsto \widetilde{\int} e^{\frac{i}{2 \hbar}\langle x,(I-L) x\rangle} e^{i\langle x, y\rangle} f(x) d x
$$

is continuous in the $\mathcal{F}(\mathcal{H})$-norm.

Let us introduce now a new type of infinite dimensional oscillatory integrals on the product space $\mathcal{H} \times \mathcal{H}$ that will be applied in the next section to the time evolution of open quantum systems. 
8 S. ALBEVERIO ${ }^{1,2), 3}$, L. CATTANEO ${ }^{1)}$, L. DI PERSIO $^{2)}$, S. MAZZUCCHI $^{2,4)}$

Definition 3. Let $f: \mathcal{H} \times \mathcal{H} \rightarrow \mathbb{C}$. If for any sequence $P_{n}$ of projectors onto $n$-dimensional subspaces of $\mathcal{H}$, such that $P_{n} \leq P_{n+1}$ and $P_{n} \rightarrow 1$ strongly as $n \rightarrow \infty$ (1 being the identity operator in $\mathcal{H})$, the finite dimensional oscillatory integrals

$$
\frac{1}{(2 \pi \hbar)^{n}} \int_{P_{n} \mathcal{H}} \int_{P_{n} \mathcal{H}} e^{\frac{i}{2 \hbar}\left\langle P_{n} x, P_{n} x\right\rangle} e^{-\frac{i}{2 \hbar}\left\langle P_{n} y, P_{n} y\right\rangle} f\left(P_{n} x, P_{n} y\right) d\left(P_{n} x\right) d\left(P_{n} y\right)
$$

are well defined and the limit

$$
\frac{1}{(2 \pi \hbar)^{n}} \int_{P_{n} \mathcal{H}} \int_{P_{n} \mathcal{H}} e^{\frac{i}{2 \hbar}\left\langle P_{n} x, P_{n} x\right\rangle} e^{-\frac{i}{2 \hbar}\left\langle P_{n} y, P_{n} y\right\rangle} f\left(P_{n} x, P_{n} y\right) d\left(P_{n} x\right) d\left(P_{n} y\right)
$$

exists and is independent of the sequence $\left\{P_{n}\right\}$, then it is denoted by:

$$
\widetilde{\iint} e^{\frac{i}{2 \hbar}\langle x, x\rangle} e^{-\frac{i}{2 \hbar}\langle y, y\rangle} f(x, y) d x d y
$$

It is possible to prove a result analogous to theorem 1

Theorem 2. Let $L: \mathcal{H} \rightarrow \mathcal{H}$ be a trace class operator, such that $I-L$ is invertible. Let $f: \mathcal{H} \times \mathcal{H} \rightarrow \mathbb{C}$ be the Fourier transform of a complex bounded variation measure $\mu_{f}$ on $\mathcal{H} \times \mathcal{H}$. Then the integral

$$
\widetilde{\int} \widetilde{\int} e^{\frac{i}{2 \hbar}\langle x, x\rangle} e^{-\frac{i}{2 \hbar}\langle y, y\rangle} e^{-\frac{i}{2 \hbar}\langle x-y, L(x+y)\rangle} f(x, y) d x d y
$$

is well defined and is equal to:

$$
\frac{1}{\operatorname{det}(I-L)} \int_{\mathcal{H}} \int_{\mathcal{H}} e^{-\frac{i \hbar}{2}\left\langle\alpha+\beta,(I-L)^{-1}(\alpha-\beta)\right\rangle} d \mu_{f}(\alpha, \beta)
$$

where $\operatorname{det}(I-L)$ is the Fredholm determinant of the operator $(I-L)$

Proof: By definition, taking a sequence $P_{n}$ of projectors onto n-dimensional subspaces of $\mathcal{H}$, such that $P_{n} \leq P_{n+1}$ and $P_{n} \rightarrow 1$ strongly as $n \rightarrow \infty$

$$
\begin{aligned}
& \widetilde{\int} \widetilde{\int} e^{\frac{i}{2 \hbar}\langle x, x\rangle} e^{-\frac{i}{2 \hbar}\langle y, y\rangle} e^{-\frac{i}{2 \hbar}\langle x-y, L(x+y)\rangle} f(x, y) d x d y \\
& =\lim _{n \rightarrow \infty} \frac{1}{(2 \pi \hbar)^{n}} \int_{P_{n} \mathcal{H}} \int_{P_{n} \mathcal{H}} e^{\frac{i}{2 \hbar}\left\langle x_{n}-y_{n},\left(I_{n}-L_{n}\right)\left(x_{n}+y_{n}\right)\right\rangle} f\left(x_{n}, y_{n}\right) d x_{n} d y_{n}
\end{aligned}
$$


where $x_{n}:=P_{n} x, x \in \mathcal{H}, I_{n}-L_{n}:=I_{\mid P_{n} \mathcal{H}}-P_{n} L P_{n}$. On the other hand, the finite dimensional approximations are defined by the following sequence of regularized integrals:

$$
\begin{aligned}
& \frac{1}{(2 \pi \hbar)^{n}} \int_{P_{n} \mathcal{H}} \int_{P_{n} \mathcal{H}} e^{\frac{i}{2 \hbar}\left\langle x_{n}-y_{n},\left(I_{n}-L_{n}\right)\left(x_{n}+y_{n}\right)\right\rangle} f\left(x_{n}, y_{n}\right) d x_{n} d y_{n} \\
= & \lim _{\epsilon \rightarrow 0} \frac{1}{(2 \pi \hbar)^{n}} \int_{P_{n} \mathcal{H}} \int_{P_{n} \mathcal{H}} e^{\frac{i}{2 \hbar}\left\langle x_{n}-y_{n},\left(I_{n}-L_{n}\right)\left(x_{n}+y_{n}\right)\right\rangle} \phi(\epsilon x, \epsilon y) f\left(x_{n}, y_{n}\right) d x_{n} d y_{n}
\end{aligned}
$$

with $\phi \in \mathcal{S}\left(\mathbb{R}^{n} \times \mathbb{R}^{n}\right), \phi(0)=1$.

By introducing the new variables $z_{n}:=x_{n}-y_{n}, w_{n}:=x_{n}+y_{n}$, by taking $n \geq \bar{n}$ and by Fubini theorem, the latter is equal to:

$$
\begin{array}{r}
\lim _{\epsilon \rightarrow 0} \frac{1}{(4 \pi \hbar)^{n}} \int_{P_{n} \mathcal{H}} \int_{P_{n} \mathcal{H}}\left(\int_{P_{n} \mathcal{H}} \int_{P_{n} \mathcal{H}} e^{i\left\langle\alpha, \frac{z+w}{2}\right\rangle+i\left\langle\beta, \frac{w-z}{2}\right\rangle}\right. \\
\left.e^{\frac{i}{2 \hbar}\left\langle z_{n},\left(I_{n}-L_{n}\right) w_{n}\right\rangle} \phi\left(\epsilon \frac{z+w}{2}, \epsilon \frac{w-z}{2}\right) d z_{n} d w_{n}\right) d \mu_{n}(\alpha, \beta) \\
=\lim _{\epsilon \rightarrow 0} \frac{\operatorname{det}\left(I_{n}-L_{n}\right)^{-1}}{(2 \pi)^{2 n}} \int_{P_{n} \mathcal{H}} \int_{P_{n} \mathcal{H}} \\
\left(\int_{P_{n} \mathcal{H}} \int_{P_{n} \mathcal{H}} e^{\frac{-i \hbar}{2}\left\langle\alpha+\beta-2 \epsilon \gamma,\left(I_{n}-L_{n}\right)^{-1}(\alpha-\beta-2 \epsilon \delta)\right\rangle}\right. \\
\left.\tilde{\phi}_{T}\left(\gamma_{n}, \delta_{n}\right) d \gamma_{n} d \delta_{n}\right) d \mu_{n}(\alpha, \beta)
\end{array}
$$

where $\mu_{n} \in \mathcal{F}\left(P_{n} \mathcal{H} \times P_{n} \mathcal{H}\right)$ is defined by: $\int_{P_{n} \mathcal{H}} \phi\left(x_{n}, y_{n}\right) d \mu_{n}\left(x_{n}, y_{n}\right):=$ $\int_{\mathcal{H}} \chi_{P_{n} \mathcal{H}}(x, y) \phi\left(P_{n} x, P_{n} y\right) d \mu(x, y)$ and $\phi_{T} \in \mathcal{S}\left(P_{n} \mathcal{H} \times P_{n} \mathcal{H}\right)$ is defined by $\phi_{T}\left(z_{n}, w_{n}\right):=\phi\left(\frac{z+w}{2}, \frac{w-z}{2}\right)$. In the third line we have used the fact that if $I-L)$ is invertible, for any sequence $\left\{P_{n}\right\}_{n \in \mathbb{N}}$ of projection operators there exist an $\bar{n}$ such that for any $n \geq \bar{n}$ the operator $P_{n}(I-$ $L) P_{N}$ is invertible, so that by taking $n$ sufficiently large $\operatorname{det}\left(I_{n}-L_{n}\right) \neq$ 0 . By applying Lebesgue's dominated convergence theorem, and by the equality

$$
\int_{P_{n} \mathcal{H}} \int_{P_{n} \mathcal{H}} \tilde{\phi}_{T}\left(\gamma_{n}, \delta_{n}\right) d \gamma_{n} d \delta_{n}=(2 \pi)^{2 n} \phi_{T}(0,0),
$$

the latter is equal to:

$$
\operatorname{det}\left(I_{n}-L_{n}\right)^{-1} \int_{P_{n} \mathcal{H}} \int_{P_{n} \mathcal{H}} e^{-\frac{i \hbar}{2}\left\langle\alpha+\beta,\left(I_{n}-L_{n}\right)^{-1}(\alpha-\beta)\right\rangle} d \mu_{n}(\alpha, \beta)
$$

By taking the limit $n \rightarrow \infty$ and by the convergence of $\operatorname{det}\left(I_{n}-L_{n}\right)$ to $\operatorname{det}(I-L)$, we get the final result

By expression (13) the next result follows easily: 
10 S. ALBEVERIO ${ }^{1), 2), 3}$, L. CATTANEO ${ }^{1)}$, L. DI PERSIO $^{2)}$, S. MAZZUCCHI ${ }^{2,4)}$

Corollary 2. Under the assumptions of theorem 2, the functional

$$
f \in \mathcal{F}(\mathcal{H} \times \mathcal{H}) \mapsto \widetilde{\int} \widetilde{\int} e^{\frac{i}{2 \hbar}\langle x, x\rangle} e^{-\frac{i}{2 \hbar}\langle y, y\rangle} e^{-i\langle x-y, L(x+y)\rangle} f(x, y) d x d y
$$

is continuous in the $\mathcal{F}(\mathcal{H} \times \mathcal{H})$-norm.

It is possible to prove the following Fubini type theorem on the change of order of integration between oscillatory integrals and Lebesgue integrals.

Let $\left\{\mu_{\alpha}: \alpha \in \mathbb{R}^{d}\right\}$ be a family in $\mathcal{M}(\mathcal{H})$. We shall let $\int_{\mathbb{R}^{d}} \mu_{\alpha} d \alpha$ denote the measure defined by

$$
\phi \mapsto \int_{\mathbb{R}^{d}} \int_{\mathcal{H}} \phi(x) d \mu_{\alpha}(x) d \alpha
$$

whenever it exists.

Theorem 3. Let $(\mathcal{H},\langle\rangle)$ and $L: \mathcal{H} \rightarrow \mathcal{H}$ as in the assumptions of theorem 2. Let $\mu: \mathbb{R}^{d} \rightarrow \mathcal{M}(\mathcal{H} \times \mathcal{H}), \alpha \mapsto \mu_{\alpha}$, be a continuous map such that

$$
\int_{\mathbb{R}^{d}}\left|\mu_{\alpha}\right| d \alpha<\infty
$$

Let $f_{\alpha}(x, y)=\hat{\mu}_{\alpha}(x, y),(x, y) \in \mathcal{H} \times \mathcal{H}$. Then $\int_{\mathbb{R}^{d}} f_{\alpha} d \alpha \in \mathcal{F}(\mathcal{H} \times \mathcal{H})$ and

$$
\begin{aligned}
& \int_{\mathbb{R}^{d}} \widetilde{\int_{\mathcal{H}}} \widetilde{\int_{\mathcal{H}}} e^{\frac{i}{2 \hbar}\langle x, x\rangle} e^{-\frac{i}{2 \hbar}\langle y, y\rangle} e^{-\frac{i}{2 \hbar}\langle x-y, L(x+y)\rangle} f_{\alpha}(x, y) d x d y d \alpha \\
& =\widetilde{\int_{\mathcal{H}}} \int_{\mathcal{H}} e^{\frac{i}{2 \hbar}\langle x, x\rangle} e^{-\frac{i}{2 \hbar}\langle y, y\rangle} e^{-\frac{i}{2 \hbar}\langle x-y, L(x+y)\rangle} \int_{\mathbb{R}^{d}} f_{\alpha}(x) d \alpha d x d y
\end{aligned}
$$

Proof: By definition of $f_{\alpha}$

$\int_{\mathbb{R}^{d}} f_{\alpha} d \alpha=\int_{\mathbb{R}^{d}} \int_{\mathcal{H} \times \mathcal{H}} e^{i\langle k, x\rangle+i\langle h, y\rangle} d \mu_{\alpha}(k, h) d \alpha=\int_{\mathcal{H} \times \mathcal{H}} e^{i\langle k, x\rangle+i\langle h, y\rangle} \int_{\mathbb{R}^{d}} d \mu_{\alpha}(k, h) d \alpha$,

so that $\int_{\mathbb{R}^{d}} f_{\alpha} d \alpha \in \mathcal{F}(\mathcal{H})$.

By applying theorem 2 to the l.h.s. of (14), we have:

$$
\begin{aligned}
& \int_{\mathbb{R}^{d}} \widetilde{\int_{\mathcal{H}}} \widetilde{\int_{\mathcal{H}}} e^{\frac{i}{2 \hbar}\langle x, x\rangle} e^{-\frac{i}{2 \hbar}\langle y, y\rangle} e^{-\frac{i}{2 \hbar}\langle x-y, L(x+y)\rangle} f_{\alpha}(x, y) d x d y d \alpha \\
& \quad=\operatorname{det}(I-L)^{-1} \int_{\mathbb{R}^{d}} \int_{\mathcal{H}} \int_{\mathcal{H}} e^{-\frac{i \hbar}{2}\left\langle k+h,(I-L)^{-1}(k-h)\right\rangle} d \mu_{\alpha}(k, h) d \alpha
\end{aligned}
$$

By the usual Fubini theorem the latter is equal to:

$$
\operatorname{det}(I-L)^{-1} \int_{\mathcal{H}} \int_{\mathcal{H}} e^{-\frac{i \hbar}{2}\left\langle k+h,(I-L)^{-1}(k-h)\right\rangle} \int_{\mathbb{R}^{d}} d \mu_{\alpha}(k, h) d \alpha
$$


that, by theorem 2 is equal to the r.h.s of of (14).

\section{The Feynman-Vernon influence functional}

The infinite dimensional oscillatory integrals of definition 2 provide a rigorous mathematical realization of the heuristic Feynman path integral representation for the solution of the Schrödinger equation. The aim of the present section is the extension of these results to the Feynman path integral representation of the time evolution of an open quantum system.

Let $U_{t}$ be the unitary evolution operator on $L^{2}\left(\mathbb{R}^{d}\right)$ whose generator is the self-adjoint extension of the operator defined on $S\left(\mathbb{R}^{d}\right)$ by $-\frac{\Delta}{2 m}+\frac{1}{2} x \Omega^{2} x+v(x)$, where $m>0, \Omega$ is a positive symmetric constant $d \times d$ matrix with eigenvalues $\Omega_{j}, j=1 \ldots d$, and $v \in \mathcal{F}\left(\mathbb{R}^{d}\right)$, $v(x)=\hat{\mu}_{v}(x)$.

The heuristic Feynman path integral representation (2) for the solution of the Schrödinger equation (1) is given by:

$\left(U(t) \psi_{0}\right)(x)=“ \widetilde{\int_{\gamma(t)=x}} e^{\frac{i}{2 \hbar}\left(m \int_{0}^{t} \dot{\gamma}(s)^{2} d s-\int_{0}^{t} \gamma(s) \Omega^{2} \gamma(s) d s\right)} e^{-\frac{i}{\hbar} \int_{0}^{t} v(\gamma(s)) d s} \phi_{0}(\gamma(0)) d \gamma$

Let us assume for notation simplicity that $m=1$ (this condition will soon be relaxed) and let us introduce the Cameron-Martin space $\mathcal{H}_{t}$, i.e. the Hilbert space of absolutely continuous paths $\gamma:[0, t] \rightarrow \mathbb{R}$, such that $\gamma(t)=0$, and square integrable weak derivative $\int_{0}^{t}|\dot{\gamma}(s)|^{2} d s<\infty$ endowed with the inner product $\left\langle\gamma_{1}, \gamma_{2}\right\rangle=\int_{0}^{t} \dot{\gamma}_{1}(s) \cdot \dot{\gamma}_{2}(s) d s$. Let $L$ : $\mathcal{H}_{t} \rightarrow \mathcal{H}_{t}$ be the trace class symmetric operator on $\mathcal{H}_{t}$ given by:

$$
(L \gamma)(s)=\int_{s}^{t} d s^{\prime} \int_{0}^{s^{\prime}} \gamma\left(s^{\prime \prime}\right) d s^{\prime \prime}, \quad \gamma \in \mathcal{H}_{t}
$$

Let $\mathcal{H}_{t}^{d}:=\oplus_{i=1}^{d} \mathcal{H}_{t}$ and let $L_{\Omega}: \mathcal{H}_{t}^{d} \rightarrow \mathcal{H}_{t}^{d}$ be the trace class symmetric operator on $\mathcal{H}_{t}^{d}$ given by:

$$
\left(L_{\Omega} \gamma\right)(s)=\int_{s}^{t} d s^{\prime} \int_{0}^{s^{\prime}}\left(\Omega^{2} \gamma\right)\left(s^{\prime \prime}\right) d s^{\prime \prime}, \quad \gamma \in \mathcal{H}_{t}^{d}
$$

One can easily verify that $\left\langle\gamma_{1}, L_{\Omega} \gamma_{2}\right\rangle=\int_{0}^{t} \gamma_{1}(s) \Omega^{2} \gamma_{2}(s) d s$. Moreover if $t \neq(n+1 / 2) \pi / \Omega_{j}, n \in \mathbb{Z}$ and $\Omega_{j}$ any eigenvalue of $\Omega,\left(I-L_{\Omega}\right)$ is 
invertible with:

$$
\begin{aligned}
\left(I-L_{\Omega}\right)^{-1} \gamma(s) & =\gamma(s)-\Omega \int_{s}^{t} \sin \left[\Omega\left(s^{\prime}-s\right)\right] \gamma\left(s^{\prime}\right) d s^{\prime}+ \\
& +\sin [\Omega(t-s)] \int_{0}^{t}[\cos \Omega t]^{-1} \Omega \cos \left(\Omega s^{\prime}\right) \gamma\left(s^{\prime}\right) d s^{\prime}
\end{aligned}
$$

and

$$
\operatorname{det}\left(I-L_{\Omega}\right)=\operatorname{det}(\cos (\Omega t))
$$

see [ET84]. Thanks to these results and under suitable assumptions it is possible to realize the heuristic Feynman path integral representation for the solution of the Schrödinger equation as a well defined infinite dimensional oscillatory integral on the Hilbert space $\mathcal{H}_{t}^{d}$.

Theorem 4. Let $\phi_{0} \in \mathcal{F}\left(\mathbb{R}^{d}\right) . t \neq(n+1 / 2) \pi / \Omega_{j}, n \in \mathbb{Z}$. Then the vector $\phi(t):=U_{t} \phi_{0}$ is given by $x \mapsto \phi(t)(x)$, with:

$$
e^{-\frac{i}{2 \hbar} x \Omega^{2} x t} \widetilde{\int_{\mathcal{H}_{t}^{d}}} e^{\frac{i}{2 \hbar}\langle\gamma,(I-L) \gamma\rangle} e^{-\frac{i}{\hbar} \int_{0}^{t} x \Omega^{2} \gamma(s) d s} e^{-\frac{i}{\hbar} \int_{0}^{t} v(\gamma(s)+x) d s} \phi_{0}(\gamma(0)+x) d \gamma
$$

For a detailed proof see [ET84].

This result can be generalized to the Feynman path integral representation of the time evolution of a mixed state:

Theorem 5. Let $\rho$ be a density matrix operator on $L^{2}\left(\mathbb{R}^{d}\right)$, such that $\rho$ admits a regular kernel $\rho(x, y), x, y \in \mathbb{R}^{d}$. Let us assume moreover that $\rho$ admits a decomposition into pure states of the form $\rho(x, y)=$ $\sum_{i} \lambda_{i} e_{i}(x) e_{i}^{*}(y)$, with $\lambda_{i}>0, \sum_{i} \lambda_{i}=1,\left\langle e_{i}, e_{j}\right\rangle_{L^{2}\left(\mathbb{R}^{d}\right)}=\delta_{i j}$, and $e_{i}(x)=$ $\hat{\mu}_{i}(x)$, satisfying:

$$
\sum_{i} \lambda_{i}\left|\mu_{i}\right|^{2}<\infty
$$

Let $t \neq(n+1 / 2) \pi / \Omega_{j}, n \in \mathbb{Z}$. Then the density matrix operator at time $t$ admits a smooth kernel $\rho_{t}(x, y)$ which is given by the infinite dimensional oscillatory integral:

$$
\begin{aligned}
& e^{-\frac{i}{2 \hbar}\left(x \Omega^{2} x-y \Omega^{2} y\right) t} \widetilde{\int_{\mathcal{H}_{t}^{m, d}}} \widetilde{\int_{\mathcal{H}_{t}^{m, d}}} e^{\frac{i}{2 \hbar}\langle\gamma,(I-L) \gamma\rangle} e^{-\frac{i}{2 \hbar}\left\langle\gamma^{\prime},(I-L) \gamma^{\prime}\right\rangle} \\
& e^{-\frac{i}{\hbar} \int_{0}^{t}\left(x \Omega^{2} \gamma(s)-y \Omega^{2} \gamma^{\prime}(s)\right) d s} e^{-\frac{i}{\hbar} \int_{0}^{t} v(\gamma(s)+x) d s} \\
& \quad e^{\frac{i}{\hbar} \int_{0}^{t} v\left(\gamma^{\prime}(s)+y\right) d s} \rho\left(\gamma(0)+x, \gamma^{\prime}(0)+y\right) d \gamma d \gamma^{\prime}
\end{aligned}
$$


Proof: By decomposing $\rho$ into pure states, by corollary 2 and condition (18) the integral (19) is equal to:

$$
\begin{aligned}
& \sum_{i} \lambda_{i}\left(e^{-\frac{i}{2 \hbar} x \Omega^{2} x t} \widetilde{\int_{\mathcal{H}_{t}^{m, d}}} e^{\frac{i}{2 \hbar}\langle\gamma,(I-L) \gamma} e^{-\frac{i}{\hbar} \int_{0}^{t} x \Omega^{2} \gamma(s) d s} e^{-\frac{i}{\hbar} \int_{0}^{t} v(\gamma(s)+x) d s} e_{i}(\gamma(0)+x) d \gamma\right) \\
&\left(e^{\frac{i}{2 \hbar} y \Omega^{2} y t} \widetilde{\int_{\mathcal{H}_{t}^{m, d}}} e^{-\frac{i}{2 \hbar}\left\langle\gamma^{\prime},(I-L) \gamma^{\prime}\right.} e^{\frac{i}{\hbar} \int_{0}^{t} y \Omega^{2} \gamma^{\prime}(s) d s} e^{\frac{i}{\hbar} \int_{0}^{t} v\left(\gamma^{\prime}(s)+y\right) d s} e_{i}^{*}(\gamma(0)+y) d \gamma\right) \\
&=\sum_{i} \lambda_{i}\left(e^{-\frac{i}{2 \hbar} x \Omega^{2} x t} \widetilde{\int_{\mathcal{H}_{t}^{m, d}}} e^{\frac{i}{2 \hbar}\langle\gamma,(I-L) \gamma} e^{-\frac{i}{\hbar} \int_{0}^{t} x \Omega^{2} \gamma(s) d s} e^{-\frac{i}{\hbar} \int_{0}^{t} v(\gamma(s)+x) d s} e_{i}(\gamma(0)+x) d \gamma\right) \\
&\left(e^{-\frac{i}{2 \hbar} y \Omega^{2} y t} \widetilde{\int_{\mathcal{H}_{t}^{m, d}}} e^{\frac{i}{2 \hbar}\left\langle\gamma^{\prime},(I-L) \gamma^{\prime}\right.} e^{-\frac{i}{\hbar} \int_{0}^{t} y \Omega^{2} \gamma^{\prime}(s) d s} e^{-\frac{i}{\hbar} \int_{0}^{t} v\left(\gamma^{\prime}(s)+y\right) d s} e_{i}(\gamma(0)+y) d \gamma\right)^{*}
\end{aligned}
$$

By theorem 5 the latter line is equal to $\sum_{i} \lambda_{i} U_{t} e_{i}(x)\left(U_{t} e_{i}\right)^{*}(y)=\rho_{t}(x, y)$.

Remark 1. Heuristically expression (19) can be written as

$$
\widetilde{\int} e^{\frac{i}{\hbar}\left(S_{t}(\gamma+x)-S_{t}\left(\gamma^{\prime}+y\right)\right.} \rho\left(\gamma(0)+x, \gamma^{\prime}(0)+y\right) d \gamma d \gamma^{\prime}
$$

where $S_{t}(\gamma)$ is the classical action of the system evaluated along the path defined in (3).

Let us consider now the time evolution of a quantum system made of two linearly interacting subsystems $A$ and $B$. Let us assume that the state space of the system $A$ is $L^{2}\left(\mathbb{R}^{d}\right)$ while the state space of the system $B$ is $L^{2}\left(\mathbb{R}^{N}\right)$. Let the total Hamiltonian of the compound systems be of the form $H_{A B}=H_{A}+H_{B}+H_{I N T}$, with $H_{A}=-\frac{\Delta_{\mathbb{R}^{d}}}{2 M}+$ $\frac{1}{2} x \Omega_{A}^{2} x+v_{A}(x), x \in \mathbb{R}^{d}, H_{B}=-\frac{\Delta_{\mathbb{R}^{N}}}{2 m}+\frac{1}{2} R \Omega_{B}^{2} R+v_{B}(R) R \in \mathbb{R}^{N}$, $H_{I N T}=x C R$, with $C: \mathbb{R}^{N} \rightarrow \mathbb{R}^{d}$ is a linear operator and $\Omega_{A}$, resp. $\Omega_{B}$, is a symmetric positive $d \times d$ (resp. $N \times N$ ) matrix. Let us assume that the quadratic part of the total potential, i.e. the function $x, R \mapsto \frac{1}{2} x \Omega_{A}^{2} x+\frac{1}{2} R \Omega_{B}^{2} R+x C R$ is positive definite (so that the total Hamiltonian is bounded from below). Let us assume moreover that the density matrix of the compound system factorizes $\rho_{A B}=\rho_{A} \rho_{B}$ and has a smooth kernel $\rho_{A B}(x, y, R, Q)=\rho_{A}(x, y) \rho_{B}(R, Q)$. We want to prove an infinite dimensional oscillatory integral representation for the reduced density operator at time $t$, namely $\int\left(U_{t} \rho_{A B} U_{t}^{+}\right)(x, y, R, R) d R$ where the unitary operator $U_{t}:=\exp \left(-\frac{1}{\hbar} H t\right)$, heuristically: 


$$
\begin{array}{r}
\widetilde{\int} \int_{\begin{array}{c}
\gamma(t)=x \\
\Gamma(t)=R
\end{array}} \widetilde{\substack{\gamma^{\prime}(t)=y \\
\Gamma^{\prime}(t)=R}} e^{\frac{i}{\hbar}\left(S_{A}(\gamma)+S_{B}(\Gamma)+S_{I N T}(\gamma, \Gamma)-S_{A}\left(\gamma^{\prime}\right)-S_{B}\left(\Gamma^{\prime}\right)-S_{I N T}\left(\gamma^{\prime}, \Gamma^{\prime}\right)\right.} \times \\
\times \rho_{A}\left(\gamma(0), \gamma^{\prime}(0)\right) \rho_{B}\left(\Gamma(0), \Gamma^{\prime}(0)\right) d \gamma d \gamma^{\prime} d \Gamma d \Gamma^{\prime} d R
\end{array}
$$

where $\gamma$ and $\Gamma$ represent the generic path in the configuration space of the system, respectively of the reservoir, and:

$$
\begin{aligned}
& S_{A}(\gamma)+S_{B}(\Gamma)+S_{I N T}(\gamma, \Gamma):=\int_{0}^{t}\left(\frac{M}{2} \dot{\gamma}^{2}(s)-\frac{1}{2} \gamma(s) \Omega_{A}^{2} \gamma(s)-v_{A}(\gamma(s)) d s\right. \\
& +\int_{0}^{t}\left(\frac{m}{2} \dot{\Gamma}^{2}(s)-\frac{1}{2} \Gamma(s) \Omega_{B}^{2} \Gamma(s)-v_{B}(\Gamma(s)) d s+\int_{0}^{t} \gamma(s) C \Gamma(s) d s\right.
\end{aligned}
$$

By the transformations in the path space, given by:

$$
\gamma \rightarrow \gamma / \sqrt{M} \text { and } \Gamma \rightarrow \Gamma / \sqrt{m}
$$

formula (21) becomes:

$$
\begin{aligned}
& \iint_{\substack{\gamma(t)=x \\
\Gamma(t)=R}} \int_{\gamma_{\gamma^{\prime}(t)=y}(t)=R} e^{\frac{i}{2 \hbar} \int_{0}^{t}\left(\dot{\gamma}^{2}(s)-\gamma(s) \frac{\Omega_{A}^{2}}{M} \gamma(s)-v_{A}\left(\frac{\gamma(s)}{M}\right) d s\right.} \times \\
\times & e^{\frac{i}{2 \hbar} \int_{0}^{t}\left(\dot{\Gamma}^{2}(s)-\Gamma(s) \frac{\Omega_{B}^{2}}{m} \Gamma(s)-v_{B}\left(\frac{\Gamma(s)}{m}\right) d s\right.} e^{-\frac{i}{\hbar} \int_{0}^{t} \gamma(s) \frac{C}{\sqrt{m M}} \Gamma(s) d s} \times \\
\times & e^{-\frac{i}{2 \hbar} \int_{0}^{t}\left(\dot{(} \gamma^{\prime}\right)^{2}(s)-\gamma^{\prime}(s) \frac{\Omega_{A}^{2}}{M} \gamma^{\prime}(s)-v_{A}\left(\frac{\gamma^{\prime}(s)}{M}\right) d s} e^{-\frac{i}{2 \hbar} \int_{0}^{t}\left(\Gamma^{\prime}\right)^{2}(s)-\Gamma^{\prime}(s) \frac{\Omega_{B}^{2}}{m} \Gamma^{\prime}(s)-v_{B}\left(\frac{\Gamma^{\prime}(s)}{m}\right) d s} \times \\
& \times e^{\frac{i}{\hbar} \int_{0}^{t} \gamma^{\prime}(s) \frac{C}{\sqrt{m M}} \Gamma^{\prime}(s) d s} \rho_{A}\left(\frac{\gamma(0)}{\sqrt{M}}, \frac{\gamma^{\prime}(0)}{\sqrt{M}}\right) \rho_{B}\left(\frac{\Gamma(0)}{\sqrt{m}}, \frac{\Gamma^{\prime}(0)}{\sqrt{m}}\right) d \gamma d \gamma^{\prime} d \Gamma d \Gamma^{\prime} d R,
\end{aligned}
$$

By transformations in (23) it is possible to take unitary masses $m$ and $M$ in order to fulfill the hypotheses of theorems 4 and 5 .

Let us consider the two Hilbert spaces:

$$
\mathcal{H}_{t}^{d}:=\underbrace{\mathcal{H}_{t} \oplus \cdots \oplus \mathcal{H}_{t}}_{d \text {-times }} \quad \text { and } \quad \mathcal{H}_{t}^{N}:=\underbrace{\mathcal{H}_{t} \oplus \cdots \oplus \mathcal{H}_{t}}_{N \text {-times }}
$$

We shall denote an element of $\mathcal{H}_{t}^{d}$, respectively of $\mathcal{H}_{t}^{N}$, by $\gamma$, respectively $\Gamma$. Let $L: \mathcal{H}_{t} \rightarrow \mathcal{H}_{t}$ be the symmetric bounded operator on $\mathcal{H}_{t}$, defined by: $L \gamma(s):=\int_{s}^{t} d s^{\prime} \int_{0}^{s^{\prime}} \gamma\left(s^{\prime \prime}\right) d s^{\prime \prime}$. Let $L_{A}: \mathcal{H}_{t}^{d} \rightarrow \mathcal{H}_{t}^{d}, L_{B}: \mathcal{H}_{t}^{N} \rightarrow \mathcal{H}_{t}^{N}$ and $L_{A B}: \mathcal{H}_{t}^{d} \oplus \mathcal{H}_{t}^{N} \rightarrow \mathcal{H}_{t}^{d} \oplus \mathcal{H}_{t}^{N}$ be the self adjoint operators defined by:

$$
L_{A} \gamma:=L^{d} \Omega_{A}^{2} M^{-1} \gamma
$$




$$
\begin{gathered}
L_{B} \Gamma:=L^{N} \Omega_{B}^{2} m^{-1} \Gamma \\
L_{A B}(\gamma, \Gamma):=\left(L_{A} \gamma+\frac{1}{\sqrt{m M}} L^{d} C \Gamma, L_{B} \Gamma+\frac{1}{\sqrt{m M}} L^{N} C^{T} \gamma\right)
\end{gathered}
$$

where, for all $k \in \mathbb{N}, L^{k}$ denotes the operator on $\mathcal{H}_{t}^{k}$ defined by:

$$
L^{k}:=L^{(1)} \otimes L^{(2)} \otimes \cdots \otimes L^{(k)}
$$

and:

$$
L^{(k)}:=\mathbf{1} \otimes \mathbf{1} \otimes \cdots \otimes \mathbf{1} \otimes \underbrace{L}_{k^{\text {th }} \text { element }} \otimes \mathbf{1} \cdots \otimes \mathbf{1}
$$

Lemma 1. Let $\Psi_{0} \in L^{2}\left(\mathbb{R}^{n+d}\right) \cap \mathcal{F}\left(\mathbb{R}^{n+d}\right)$ and let $v_{A} \in \mathcal{F}\left(\mathbb{R}^{d}\right), v_{B} \in$ $\mathcal{F}\left(\mathbb{R}^{N}\right)$. Let $t \neq(n+1 / 2) \pi / \lambda_{j}$, where $n \in \mathbb{Z}$ and $\lambda_{j}^{2}, j=1, \ldots d+N$, are the eigenvalues of the matrix:

$$
\left(\begin{array}{cc}
\Omega_{A}^{\prime 2} & C^{\prime} \\
C^{\prime T} & \Omega_{B}^{\prime 2}
\end{array}\right) \quad \Omega_{A}^{\prime}:=\Omega_{A} / \sqrt{M}, \Omega_{B}^{\prime}:=\Omega_{B} / \sqrt{m}, C^{\prime}:=C / \sqrt{M m}
$$

Then the solution of the Schrödinger equation evaluated at time t:

$$
\left\{\begin{array}{l}
i \hbar \frac{\partial}{\partial t} \Psi=H_{A B} \psi \\
\Psi(0, x, R)=\Psi_{0}(x, R), \quad(x, R) \in \mathbb{R}^{d} \times \mathbb{R}^{N}
\end{array}\right.
$$

is a smooth function and is represented by the infinite dimensional oscillatory integral:



where we have defined the functions:

$$
\Psi_{0}^{\prime}(x, R):=\Psi_{0}(x / \sqrt{M}, R / \sqrt{m})
$$

and:

$$
\begin{aligned}
& G(\gamma, \Gamma, x, R):=e^{-\frac{i t}{2 \hbar} x \Omega_{A}^{\prime 2} x-\frac{i t}{2 \hbar} R \Omega_{B}^{\prime 2} R-\frac{i}{\hbar} x C^{\prime} R t} \times \\
& \times e^{-\frac{i}{\hbar} \int_{0}^{t} x \Omega_{A}^{\prime 2} \gamma(s) d s-\frac{i}{\hbar} \int_{0}^{t} R \Omega_{B}^{\prime 2} \Gamma(s) d s-\frac{i}{\hbar} \int_{0}^{t} x C^{\prime} \Gamma(s) d s-\frac{i}{\hbar} \int_{0}^{t} \gamma(s) C^{\prime} R d s} \times \\
& \times e^{-\frac{i}{\hbar} \int_{0}^{t} v_{A}^{\prime}(\gamma(s)+x) d s-\frac{i}{\hbar} \int_{0}^{t} v_{B}^{\prime}(\Gamma(s)+x) d s}
\end{aligned}
$$

while $v_{A}^{\prime}$ and $v_{B}^{\prime}$ are defined as follows:

$$
v_{A}^{\prime}(x):=v_{A}(x / \sqrt{M}) ; v_{B}^{\prime}(R):=v_{B}(R / \sqrt{m})
$$

Proof: Let $\xi_{1}, \ldots \xi_{d+N}$ be a system of normal coordinates in $\mathbb{R}^{d+N}$, with $(x, R)=U\left(\xi_{1}, \ldots \xi_{d+N}\right), U^{T}=U^{-1}$, the quadratic part of the action is diagonalized and it is possible to apply theorem 4 . The result follows 
16 S. ALBEVERIO ${ }^{1), 2), 3}$, L. CATTANEO ${ }^{1)}$, L. DI PERSIO ${ }^{2)}$, S. MAZZUCCHI ${ }^{2,4)}$

by the invariance of the infinite dimensional oscillatory integrals under unitary transformation on paths space [AHK76], and by the infinite dimensional oscillatory integral representation for the solution of the Schrödinger equation with a potential of the type " harmonic oscillator plus Fourier transform of measure" (see [ABHK82, ET84, AB93] for more details).

Lemma 2. Let $f \in \mathcal{F}\left(\mathcal{H}_{t}^{d} \oplus \mathcal{H}_{t}^{N}\right), f=\hat{\mu}$. Let $t$ satisfy the following inequalities

$$
\begin{gathered}
t \neq(n+1 / 2) \pi / \Omega_{j}^{A}, \quad n \in \mathbb{Z}, \quad j=1 \ldots d, \\
t \neq(n+1 / 2) \pi / \Omega_{j}^{B}, \quad n \in \mathbb{Z}, \quad j=1 \ldots N, \\
t \neq(n+1 / 2) \pi / \lambda_{j}, \quad n \in \mathbb{Z}, \quad j=1 \ldots d+N,
\end{gathered}
$$

where $\Omega_{j}^{A}, j=1 \ldots d, \Omega_{j}^{B}, j=1 \ldots N$, and $\lambda_{j}, j=1 \ldots d+N$ are respectively the eigenvalues of the matrices $\Omega_{A}^{\prime}, \Omega_{B}^{\prime}$ and of the matrix given by (28). Let $L_{A}, L_{B}, L_{A B}$ be defined respectively by (25), (26) and (27). Then the function:

$$
\gamma \in \mathcal{H}_{t}^{d} \mapsto \widetilde{\int_{\mathcal{H}_{t}^{N}}} e^{\frac{i}{2 \hbar}\left\langle\Gamma,\left(I_{N}-L_{B}\right) \Gamma\right\rangle} e^{-\frac{i}{\hbar}\left\langle\Gamma, L^{N} C^{\prime T} \gamma\right\rangle} f(\gamma, \Gamma) d \Gamma
$$

is Fresnel integrable and:

$$
\begin{aligned}
& \widetilde{\int_{\mathcal{H}_{t}^{d} \oplus \mathcal{H}_{t}^{N}}} e^{\frac{i}{2 \hbar}\left\langle(\gamma, \Gamma),\left(I_{d+N}-L_{A B}\right)(\gamma, \Gamma)\right\rangle} f(\gamma, \Gamma) d \gamma d \Gamma= \\
& =\int_{\mathcal{H}_{t}^{d}} e^{\frac{i}{2 \hbar}\left\langle\gamma,\left(I_{d}-L_{A}\right) \gamma\right\rangle}\left(\widetilde{\int_{\mathcal{H}_{t}^{N}}} e^{\frac{i}{2 \hbar}\left\langle\Gamma,\left(I_{N}-L_{B}\right) \Gamma\right\rangle} e^{-\frac{i}{\hbar}\left\langle\Gamma, L^{N} C^{\prime T} \gamma\right\rangle} f(\gamma, \Gamma) d \Gamma\right) d \gamma
\end{aligned}
$$

Proof: By condition (33) the operator $I_{N}-L_{B}$ is invertible and by theorem 1 we have:

$$
\begin{gathered}
\widetilde{\int_{\mathcal{H}_{t}^{N}}} e^{\frac{i}{2 \hbar}\left\langle\Gamma,\left(I_{N}-L_{B}\right) \Gamma\right\rangle} e^{-\frac{i}{\hbar}\left\langle\Gamma, L^{N} C^{\prime T} \gamma\right\rangle} f(\gamma, \Gamma) d \Gamma \\
=\operatorname{det}\left(I_{N}-L_{B}\right)^{-1 / 2} \int_{\mathcal{H}_{t}^{N}} e^{-\frac{i \hbar}{2}\left\langle\Gamma-\frac{L^{N} C^{\prime T} \gamma}{\hbar},\left(I_{N}-L_{B}\right)^{-1} \Gamma-\frac{L^{N} C^{\prime T} \gamma}{\hbar}\right\rangle} d \mu_{\gamma}(\Gamma) \\
=\operatorname{det}\left(I_{N}-L_{B}\right)^{-1 / 2} e^{-\frac{i}{2 \hbar}\left\langle\gamma, C^{\prime} L^{N}\left(I_{N}-L_{B}\right)^{-1} L^{N} C^{\prime T} \gamma\right\rangle} \\
\int_{\mathcal{H}_{t}^{N}} e^{-\frac{i \hbar}{2}\left\langle\Gamma,\left(I_{N}-L_{B}\right)^{-1} \Gamma\right\rangle} e^{i\left\langle\gamma, C^{\prime} L^{N}\left(I_{N}-L_{B}\right)^{-1} \Gamma\right\rangle} d \mu_{\gamma}(\Gamma)
\end{gathered}
$$


where $\mu_{\gamma}$ is the measure on $\mathcal{H}_{t}^{N}$ defined by:

$$
\int_{\mathcal{H}_{t}^{N}} g(\Gamma) d \mu_{\gamma}(\Gamma):=\int_{\mathcal{H}_{t}^{d} \times \mathcal{H}_{t}^{N}} g(\Gamma) e^{i\left\langle\gamma, \gamma^{\prime}\right\rangle} d \mu\left(\gamma^{\prime}, \Gamma\right) .
$$

One can also easily verify that the operator on $\mathcal{H}_{t}^{d}$ defined by:

$$
\gamma \mapsto\left(L_{A}+C^{\prime} L^{N}\left(I_{N}-L_{B}\right)^{-1} L^{N} C^{T}\right) \gamma
$$

is trace class and, if conditions (32),(32) and (34) are satisfied, the operator defined by:

$$
\gamma \mapsto\left(I_{d}-L_{A}+C^{\prime} L^{N}\left(I_{N}-L_{B}\right)^{-1} L^{N} C^{T}\right) \gamma
$$

is invertible. Moreover the function defined by

$$
\gamma \mapsto \int_{\mathcal{H}_{t}^{N}} e^{-\frac{i \hbar}{2}\left\langle\Gamma,\left(I_{N}-L_{B}\right)^{-1} \Gamma\right\rangle} e^{i\left\langle\gamma, C^{\prime} L^{N}\left(I_{N}-L_{B}\right)^{-1} \Gamma\right\rangle} d \mu_{\gamma}(\Gamma)
$$

is the Fourier transform of the bounded variation measure $\nu$ on $\mathcal{H}_{t}$ defined by

$$
\int_{\mathcal{H}_{t}^{d}} g(\gamma) d \nu(\gamma):=\int_{\mathcal{H}_{t}^{d} \times \mathcal{H}_{t}^{N}} g\left(\gamma+C^{\prime} L^{N}\left(I_{N}-L_{B}\right)^{-1} \Gamma\right) e^{-\frac{i \hbar}{2}\left\langle\Gamma,\left(I_{N}-L_{B}\right)^{-1} \Gamma\right\rangle} d \mu(\gamma, \Gamma)
$$

By applying theorem 1 we have:

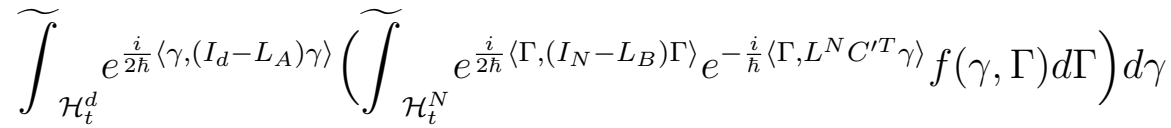

$$
\begin{aligned}
& =\operatorname{det}\left(I_{d}-L_{A}-C^{\prime} L^{N}\left(I_{N}-L_{B}\right)^{-1} L^{N} C^{T}\right)^{-1 / 2} \operatorname{det}\left(I_{N}-L_{B}\right)^{-1 / 2} \\
& \int_{\mathcal{H}_{t}^{d} \oplus \mathcal{H}_{t}^{N}} e^{-\frac{i \hbar}{2}\left\langle\gamma+C^{\prime} L^{N}\left(I_{N}-L_{B}\right)^{-1} \Gamma,\left(I_{d}-L_{A}-C^{\prime} L^{N}\left(I_{N}-L_{B}\right)^{-1} L^{N} C^{\prime T}\right)^{-1}\left(\gamma+C^{\prime} L^{N}\left(I_{N}-L_{B}\right)^{-1} \Gamma\right)\right\rangle} \\
& e^{-\frac{i \hbar}{2}\left\langle\Gamma,\left(I_{N}-L_{B}\right)^{-1} \Gamma\right\rangle} d \mu(\gamma, \Gamma)
\end{aligned}
$$

On the other hand the oscillatory integral:

$$
\widetilde{\int_{\mathcal{H}_{t}^{d} \oplus \mathcal{H}_{t}^{N}}} e^{\frac{i}{2 \hbar}\left\langle(\gamma, \Gamma),\left(I_{d+N}-L_{A B}\right)(\gamma, \Gamma)\right\rangle} f(\gamma, \Gamma) d \gamma d \Gamma
$$

is equal, again by theorem 1, to:

$$
\operatorname{det}\left(I-L_{A B}\right)^{-1 / 2} \int_{\mathcal{H}_{t}^{d} \oplus \mathcal{H}_{t}^{N}} e^{-\frac{i \hbar}{2}\left\langle(\gamma, \Gamma),\left(I_{d+N}-L_{A B}\right)^{-1}(\gamma, \Gamma)\right\rangle} d \mu(\gamma, \Gamma)
$$

Where $L_{A B}$ is defined by (27), so that an element $\left(\gamma^{\prime}, \Gamma^{\prime}\right) \in \mathcal{H}_{t}^{d} \oplus \mathcal{H}_{t}^{N}$ is equal to $\left(I_{d+N}-L_{A B}\right)^{-1}(\gamma, \Gamma),(\gamma, \Gamma) \in \mathcal{H}_{t}^{d} \oplus \mathcal{H}_{t}^{N}$ if and only if

$$
\left\{\begin{array}{l}
\left(I_{d}-L_{A}\right) \gamma^{\prime}-L^{d} C^{\prime} \Gamma^{\prime}=\gamma \\
\left(I_{N}-L_{B}\right) \Gamma^{\prime}-L^{N} C^{\prime T} \gamma^{\prime}=\Gamma
\end{array}\right.
$$


and one can easily verify that the solution is:

$$
\begin{gathered}
\gamma^{\prime}=\left(I_{d}-L_{A}-C^{\prime} L^{N}\left(I_{N}-L_{B}\right)^{-1} L^{N} C^{T}\right)^{-1} \gamma+ \\
+\left(I_{d}-L_{A}\right)^{-1} L^{d} C^{\prime}\left(I_{N}-L_{B}-L^{N} C^{\prime}\left(I_{d}-L_{A}\right)^{-1} L^{d} C^{\prime}\right)^{-1} \Gamma \\
\Gamma^{\prime}=\left(I_{N}-L_{B}\right)^{-1} L_{N} C^{\prime T}\left(I_{d}-L_{A}-L^{d} C^{\prime}\left(I_{N}-L_{B}\right)^{-1} L_{N} C^{\prime T}\right)^{-1} \gamma+ \\
\quad+\left(I_{N}-L_{B}-L^{N} C^{\prime T}\left(I_{d}-L_{A}\right)^{-1} L^{d} C^{\prime}\right)^{-1} \Gamma
\end{gathered}
$$

As a consequence the exponent in the integral (38) is equal to:

$$
\begin{gathered}
\left\langle(\gamma, \Gamma),\left(I_{d+N}-L_{A B}\right)^{-1}(\gamma, \Gamma)\right\rangle_{\mathcal{H}_{t}^{d} \oplus \mathcal{H}_{t}^{N}}= \\
=\left\langle\gamma,\left(I_{d}-L_{A}-C^{\prime} L^{N}\left(I_{N}-L_{B}\right)^{-1} L^{N} C^{T}\right)^{-1} \gamma\right\rangle_{\mathcal{H}_{t}^{d}} \\
+\left\langle\gamma,\left(I_{d}-L_{A}\right)^{-1} L^{d} C^{\prime}\left(I_{N}-L_{B}-L^{N} C^{\prime}\left(I_{d}-L_{A}\right)^{-1} L^{d} C^{\prime}\right)^{-1} \Gamma\right\rangle_{\mathcal{H}_{t}^{d}}+ \\
\quad+\left\langle\Gamma,\left(I_{N}-L_{B}-L^{N} C^{\prime T}\left(I_{d}-L_{A}\right)^{-1} L^{d} C^{\prime}\right)^{-1} \Gamma\right\rangle_{\mathcal{H}_{t}^{N}} \\
+\left\langle\Gamma,\left(I_{N}-L_{B}\right)^{-1} L_{N} C^{\prime T}\left(I_{d}-L_{A}-L^{d} C^{\prime}\left(I_{N}-L_{B}\right)^{-1} L_{N} C^{\prime T}\right)^{-1} \gamma\right\rangle_{\mathcal{H}_{t}^{N}}
\end{gathered}
$$

As one can easily verify that:

$$
\begin{aligned}
& \left(I_{N}-L_{B}-L^{N} C^{\prime T}\left(I_{d}-L_{A}\right)^{-1} L^{d} C^{\prime}\right)^{-1}=\left(I_{N}-L_{B}\right)^{-1} \\
+ & \left(I_{N}-L_{B}\right)^{-1} L^{N} C^{\prime T}\left(I-L_{A}-C^{\prime} L^{N}\left(I_{N}-L_{B}\right)^{-1} L^{N} C^{\prime T}\right)^{-1} C^{\prime} L^{N}\left(I-L_{B}\right)^{-1},
\end{aligned}
$$

and analogously:

$$
\begin{aligned}
& \left(I_{d}-L_{A}-C^{\prime} L^{N}\left(I_{N}-L_{B}\right)^{-1} L^{N} C^{T}\right)^{-1} C^{\prime} L^{N}\left(I_{N}-L_{B}\right)^{-1} \\
& \quad=\left(I_{d}-L_{A}\right)^{-1} L^{d} C^{\prime}\left(I_{N}-L_{B}-L^{N} C^{T}\left(I_{d}-L_{A}\right)^{-1} L^{d} C^{\prime}\right)^{-1}
\end{aligned}
$$

from which we conclude that the integral (38) is equal to the integral (37).

Equality (35) follows by the following relation:

$\operatorname{det}\left(I-L_{A B}\right)=\operatorname{det}\left(I_{d}-L_{A}-C^{\prime} L^{N}\left(I_{N}-L_{B}\right)^{-1} L^{N} C^{\prime T}\right) \operatorname{det}\left(I_{N}-L_{B}\right)$,

that can be verified by writing the operator $I_{d+N}-L_{A B}$ in block form, i.e. $I_{d+N}-L_{A B}=\left(\begin{array}{cc}I_{N}-L_{B} & L^{N} C^{\prime T} \\ L^{d} C^{\prime} & I_{d}-L_{A}\end{array}\right)$, by taking the finite dimensional approximation of both sides of equation (42) and by the analogous equality valid for finite dimensional matrices. 
Lemma 3. Let $\psi_{0}^{A} \in L^{2}\left(\mathbb{R}^{d}\right) \cap \mathcal{F}\left(\mathbb{R}^{d}\right), \psi_{0}^{B} \in L^{2}\left(\mathbb{R}^{N}\right) \cap \mathcal{F}\left(\mathbb{R}^{N}\right)$. Let $t$ satisfy assumptions (32),(33) and (34). Then the solution of the Schrödinger equation (1) is equal to:

$$
\begin{aligned}
& \widetilde{\int_{\mathcal{H}_{t}^{d}}} e^{\frac{i}{2 \hbar}\left\langle\gamma,\left(I_{d}-L_{A}\right) \gamma\right\rangle}\left(\widetilde{\int_{\mathcal{H}_{t}^{N}}} e^{\frac{i}{2 \hbar}\left\langle\Gamma,\left(I_{N}-L_{B}\right) \Gamma\right\rangle} e^{-\frac{i}{\hbar}\left\langle\Gamma, L^{N} C^{\prime T} \gamma\right\rangle} \times\right. \\
& \left.\times G(\gamma, \Gamma, x, R) \psi_{0, A}^{\prime}(\gamma(0)+x) \psi_{0, B}^{\prime}(\Gamma(0)+R) d \Gamma\right) d \gamma
\end{aligned}
$$

where $G(\gamma, \Gamma, x, R)$ is given by $(31)$ and $\psi_{0, A}^{\prime}(x):=\psi_{0}^{A}(x / \sqrt{M}), \psi_{0, B}^{\prime}(R):=$ $\psi_{0}^{B}(R / \sqrt{m})$.

Proof: The result follows by lemma 1 and lemma 2 with $\psi_{0}=\psi_{0}^{A} \otimes \psi_{0}^{B}$.

Theorem 6. Let $\rho_{0}^{A}$ and $\rho_{0}^{B}$ be two density matrix operators on $L^{2}\left(\mathbb{R}^{d}\right)$ and $L^{2}\left(\mathbb{R}^{N}\right)$ respectively. Let us assume that they have smooth kernels, denoted by $\rho_{0}^{A}\left(x, x^{\prime}\right)$ and $\rho_{0}^{B}\left(R, R^{\prime}\right)$. Let us assume moreover that they decompose into sum of pure states

$$
\rho_{0}^{A}=\sum_{i} w_{i}^{A} P_{\psi_{i}^{A}}, \quad \rho_{0}^{B}=\sum_{j} w_{j}^{B} P_{\psi_{j}^{B}}, \quad \psi_{i}^{A}=\hat{\mu}_{i}^{A}, \psi_{j}^{B}=\hat{\mu}_{j}^{B}
$$

with $\mu_{i}^{A} \in \mathcal{F}\left(\mathbb{R}^{d}\right), \mu_{j}^{B} \in \mathcal{F}\left(\mathbb{R}^{N}\right)$, and:

$$
\sum_{i, j} w_{i}^{A} w_{j}^{B}\left|\mu_{i}^{A}\right|^{2}\left|\mu_{j}^{B}\right|^{2}<+\infty
$$

Let $t$ satisfy assumptions (32), (33), (34).

Then the kernel $\rho_{t}\left(x, x^{\prime}, R, R^{\prime}\right)$ of the density operator of the system evaluated at time $t$ is given by the following infinite dimensional oscillatory integral (in the sense of definition 3):

$$
\begin{array}{r}
\widetilde{\int_{\mathcal{H}_{t}^{d} \oplus \mathcal{H}_{t}^{N}}} \widetilde{\mathcal{H}_{t}^{d} \oplus \mathcal{H}_{t}^{N}} e^{\frac{i}{2 \hbar}\left\langle(\gamma, \Gamma),\left(I_{d+N}-L_{A B}\right)(\gamma, \Gamma)\right\rangle} e^{-\frac{i}{2 \hbar}\left\langle\left(\gamma^{\prime}, \Gamma^{\prime}\right),\left(I_{d+N}-L_{A B}\right)\left(\gamma^{\prime}, \Gamma^{\prime}\right)\right\rangle} \\
G(\gamma, \Gamma, x, R) \bar{G}\left(\gamma^{\prime}, \Gamma^{\prime}, x^{\prime}, R^{\prime}\right) \rho_{0, A}^{\prime}\left(\gamma(0)+x, \gamma^{\prime}(0)+x^{\prime}\right) \\
\rho_{0, B}^{\prime}\left(\Gamma(0)+R, \Gamma^{\prime}(0)+R^{\prime}\right) d \gamma d \Gamma d \gamma^{\prime} d \Gamma^{\prime}
\end{array}
$$


where $G(\gamma, \Gamma, x, R)$ is given by (31). It is also equal to:

$$
\begin{gathered}
\widetilde{\int_{\mathcal{H}_{t}^{d}}} \widetilde{\int}_{\mathcal{H}_{t}^{d}} e^{\frac{i}{2 \hbar}\left\langle\gamma,\left(I_{d}-L_{A}\right) \gamma\right\rangle} e^{-\frac{i}{2 \hbar}\left\langle\gamma^{\prime},\left(I_{d}-L_{A}\right) \gamma^{\prime}\right\rangle} \widetilde{\int_{\mathcal{H}_{t}^{N}}} \widetilde{\int}_{\mathcal{H}_{t}^{N}} e^{\frac{i}{2 \hbar}\left\langle\Gamma,\left(I_{N}-L_{B}\right) \Gamma\right\rangle} \\
e^{-\frac{i}{\hbar}\left\langle\Gamma, L^{N} C^{T} \gamma\right\rangle} e^{-\frac{i}{2 \hbar}\left\langle\Gamma^{\prime},\left(I_{N}-L_{B}\right) \Gamma^{\prime}\right\rangle} e^{\frac{i}{\hbar}\left\langle\Gamma^{\prime}, L^{N} C^{T} \gamma^{\prime}\right\rangle} G(\gamma, \Gamma, x, R) \bar{G}\left(\gamma^{\prime}, \Gamma^{\prime}, x^{\prime}, R^{\prime}\right) \\
\left.\rho_{0, B}^{\prime}\left(\Gamma(0)+R, \Gamma^{\prime}(0)+R^{\prime}\right) d \Gamma d \Gamma^{\prime}\right) \rho_{0, A}^{\prime}\left(\gamma(0)+x, \gamma^{\prime}(0)+x^{\prime}\right) d \gamma d \gamma^{\prime}
\end{gathered}
$$

where $\rho_{0, A}^{\prime}(x, y):=\rho_{0}^{A}(x / \sqrt{M}, y / \sqrt{M})$ and $\rho_{0, B}^{\prime}(R, Q):=\rho_{0}^{B}(R / \sqrt{m}, Q / \sqrt{m})$.

Proof: If $\rho_{0}^{A}$ and $\rho_{0}^{B}$ are pure states, the result is a direct consequence of lemma 1 and lemma 3 .

For general $\rho_{0}^{A}$ and $\rho_{0}^{B}$ satisfying assumptions (44) and (45) the result follows by the continuity of the infinite dimensional oscillatory integral as a functional of $\mathcal{F}\left(\mathbb{R}^{N+d}\right)$ (corollary 2$)$.

Theorem 7. Let $\rho_{0}^{A}$ and $\rho_{0}^{B}$ be two density matrix operators on $L^{2}\left(\mathbb{R}^{d}\right)$ and $L^{2}\left(\mathbb{R}^{N}\right)$ respectively. Let us assume that they have regular kernels as assumed in theorem 5 , denoted by $\rho_{0}^{A}\left(x, x^{\prime}\right)$ and $\rho_{0}^{B}\left(R, R^{\prime}\right)$. Let $\rho_{0}^{B} \in$ $S\left(\mathbb{R}^{N} \times \mathbb{R}^{N}\right)$. Let us assume that $t$ satisfies assumptions (32), (33), (34) and that $t$ is such that the determinant of the $d \times d$ left upper block of the $n \times n$ matrix $\cos (\Omega t), \Omega^{2}$ being the matrix (28), is non vanishing. Then the kernel $\rho_{R}(t, x, y)$ of the reduced density operator of the system $A$ evaluated at time $t$ is given by:

$$
\begin{array}{r}
\rho_{R}(t, x, y)=e^{-\frac{i t}{2 \hbar} x \Omega_{A}^{\prime 2} x} e^{\frac{i t}{2 \hbar} y \Omega_{A}^{\prime 2} y} \widetilde{\int_{\mathcal{H}_{t}^{d}}} \widetilde{\mathcal{H}_{t}^{d}} e^{\frac{i}{2 \hbar}\left\langle\gamma,\left(I_{d}-L_{A}\right) \gamma\right\rangle} e^{-\frac{i}{2 \hbar}\left\langle\gamma^{\prime},\left(I_{d}-L_{A}\right) \gamma^{\prime}\right\rangle} \\
e^{-\frac{i}{\hbar} \int_{0}^{t} x \Omega_{A}^{\prime 2} \gamma(s) d s} e^{\frac{i}{\hbar} \int_{0}^{t} y \Omega_{A}^{\prime 2} \gamma^{\prime}(s) d s} e^{-\frac{i}{\hbar} \int_{0}^{t} v_{A}^{\prime}(\gamma(s)+x) d s+\frac{i}{\hbar} \int_{0}^{t} v_{A}^{\prime}\left(\gamma^{\prime}(s)+y\right) d s} \\
F\left(\gamma, \gamma^{\prime}, x, y\right) \rho_{0, A}^{\prime}\left(\gamma(0)+x, \gamma^{\prime}(0)+y\right) d \gamma d \gamma^{\prime}
\end{array}
$$

where $F\left(\gamma, \gamma^{\prime}, x, y\right)$ is the influence functional is given by:

$$
\begin{gathered}
F\left(\gamma, \gamma^{\prime}, x, y\right):=\int_{\mathbb{R}^{N}} e^{-\frac{i t}{\hbar} x C^{\prime} R} e^{+\frac{i t}{\hbar} y C^{\prime} R} e^{-\frac{i}{\hbar} \int_{0}^{t}\left(\gamma(s)-\gamma^{\prime}(s)\right) C^{\prime} R d s} \\
\widetilde{\int_{\mathcal{H}_{t}^{N}}} \widetilde{\mathcal{H}_{t}^{N}} e^{\frac{i}{2 \hbar}\left\langle\Gamma,\left(I_{N}-L_{B}\right) \Gamma\right\rangle} e^{-\frac{i}{2 \hbar}\left\langle\Gamma^{\prime},\left(I_{N}-L_{B}\right) \Gamma^{\prime}\right\rangle} e^{-\frac{i}{\hbar}\left\langle\Gamma, L^{N} C^{\prime T} \gamma\right\rangle} e^{\frac{i}{\hbar}\left\langle\Gamma^{\prime}, L^{N} C^{\prime T} \gamma^{\prime}\right\rangle} \\
e^{-\frac{i}{\hbar} \int_{0}^{t} R \Omega_{B}^{2}\left(\Gamma(s)-\Gamma^{\prime}(s)\right) d s} e^{-\frac{i}{\hbar} \int_{0}^{t}\left(x C^{\prime} \Gamma(s)+y C^{\prime} \Gamma^{\prime}(s)\right) d s} \\
e^{-\frac{i}{\hbar} \int_{0}^{t} v_{B}^{\prime}(\Gamma(s)+R) d s+\frac{i}{\hbar} \int_{0}^{t} v_{B}^{\prime}\left(\Gamma^{\prime}(s)+R\right) d s} \rho_{0, B}^{\prime}\left(\Gamma(0)+R, \Gamma^{\prime}(0)+R\right) d \Gamma d \Gamma^{\prime} d R
\end{gathered}
$$


Proof:

Let us assume for notation simplicity that $m=M=1$. The result in the general case can be obtained by replacing $\Omega_{A}, \Omega_{B}, C, v_{A}, v_{B}, \rho_{0}^{A}, \rho_{0}^{B}$ by $\Omega_{A}^{\prime}, \Omega_{B}^{\prime}, C^{\prime}, v_{A}^{\prime}, v_{B}^{\prime}, \rho_{0, A}^{\prime}, \rho_{0, B}^{\prime}$.

First step: Let us prove first of all that the functional $\left(\gamma \gamma^{\prime}\right) \mapsto F\left(\gamma, \gamma^{\prime}, x, y\right)$ is well defined for any $\gamma, \gamma^{\prime} \in \mathcal{H}_{t}^{d}, x, y \in \mathbb{R}^{d}$ and it is Fresnel integrable in the sense of definition 3 .

By decomposing the mixed state $\rho_{0}^{B}$ into pure states according to the formula (44), the influence functional can be written as:

$$
\int_{\mathbb{R}^{N}} \sum_{j} w_{j}^{B} \psi_{j}^{B}(x, \gamma ; R) \psi_{j}^{B}\left(y, \gamma^{\prime} ; R\right) d R
$$

where $\psi_{j}^{B}(x, \gamma)$ is the solution of the Schrödinger equation with initial datum $\psi_{j}^{B}$ and Hamiltonian $H=-\frac{1}{2} \Delta_{R}+\frac{1}{2} R \Omega^{2} B R+(x+\gamma(t)) C R+$ $v_{B}(R)$. In particular, by the unitarity of the evolution operator, $\left\|\psi_{j}^{B}(x, \gamma)\right\|_{L^{2}\left(\mathbb{R}^{N}\right)}=$ 1 for any $x \in \mathbb{R}^{d} \gamma \in \mathcal{H}_{t}^{d}$. As, by Schwartz inequality:

$$
\begin{aligned}
\sum_{j} w_{j}^{B} \int_{\mathbb{R}^{N}} \psi_{j}^{B}(x, \gamma ; R) \psi_{j}^{B}\left(y, \gamma^{\prime} ; R\right) d R \\
\quad \leq \sum_{j} w_{j}^{B}\left\|\psi_{j}^{B}(x, \gamma)\right\|_{L^{2}\left(\mathbb{R}^{N}\right)}\left\|\psi_{j}^{B}\left(y, \gamma^{\prime}\right)\right\|_{L^{2}\left(\mathbb{R}^{N}\right)}=1
\end{aligned}
$$

we can conclude that $F\left(\gamma, \gamma^{\prime}, x, y\right)$ is well defined for any $x, y \in \mathbb{R}^{d}$ $\gamma, \gamma^{\prime} \in \mathcal{H}_{t}^{d}$. Moreover, by Lebesgue's dominated convergence theorem, we have:

$$
\begin{gathered}
F\left(\gamma, \gamma^{\prime}, x, y\right)=\lim _{\epsilon \rightarrow 0^{+}} \int_{\mathbb{R}^{N}} e^{-\epsilon R^{2}} e^{-\frac{i t}{\hbar} x C R} e^{+\frac{i t}{\hbar} y C R} e^{-\frac{i}{\hbar} \int_{0}^{t}\left(\gamma(s)-\gamma^{\prime}(s)\right) C R d s} \\
\widetilde{\int_{\mathcal{H}_{t}^{N}}} \widetilde{\mathcal{H}_{t}^{N}} e^{\frac{i}{2 \hbar}\left\langle\Gamma,\left(I_{N}-L_{B}\right) \Gamma\right\rangle} e^{-\frac{i}{2 \hbar}\left\langle\Gamma^{\prime},\left(I_{N}-L_{B}\right) \Gamma^{\prime}\right\rangle} e^{-\frac{i}{\hbar}\left\langle\Gamma, L^{N} C^{T} \gamma\right\rangle} e^{\frac{i}{\hbar}\left\langle\Gamma^{\prime}, L^{N} C^{T} \gamma^{\prime}\right\rangle} \\
e^{-\frac{i}{\hbar} \int_{0}^{t} R \Omega_{B}^{2}\left(\Gamma(s)-\Gamma^{\prime}(s)\right) d s} e^{-\frac{i}{\hbar} \int_{0}^{t}\left(x C \Gamma(s)+y C \Gamma^{\prime}(s)\right) d s} \\
e^{-\frac{i}{\hbar} \int_{0}^{t} v_{B}(\Gamma(s)+R) d s+\frac{i}{\hbar} \int_{0}^{t} v_{B}\left(\Gamma^{\prime}(s)+R\right) d s} \rho_{0}^{B}\left(\Gamma(0)+R, \Gamma^{\prime}(0)+R\right) d \Gamma d \Gamma^{\prime} d R
\end{gathered}
$$


By theorem 2 we have:

$$
\begin{aligned}
& F\left(\gamma, \gamma^{\prime}, x, y\right)=\left|\operatorname{det}\left(I_{N}-L_{B}\right)\right|^{-1} \lim _{\epsilon \rightarrow 0^{+}} \int_{\mathbb{R}^{N}} d R e^{-\epsilon R^{2}} e^{-\frac{i t}{\hbar} x C R} \\
& e^{+\frac{i t}{\hbar} y C R} e^{-\frac{i}{\hbar} \int_{0}^{t}\left(\gamma(s)-\gamma^{\prime}(s)\right) C R d s} \sum_{n=0}^{\infty} \sum_{m=0}^{\infty} \frac{1}{n !} \frac{1}{m !}\left(\frac{-i}{\hbar}\right)^{n}\left(\frac{i}{\hbar}\right)^{m} \\
& \int_{0}^{t} \ldots \int_{0}^{t} \int_{0}^{t} \ldots \int_{0}^{t} \prod_{i=1}^{n} d s_{i} \prod_{j=1}^{m} d r_{j} \int_{\mathbb{R}^{N}} \ldots \int_{\mathbb{R}^{N}} \int_{\mathbb{R}^{N}} \ldots \int_{\mathbb{R}^{N}} \prod_{i=1}^{n} d \mu_{v}\left(k_{i}\right) \prod_{j=1}^{m} d \mu_{v}\left(h_{j}\right) \\
& \int_{\mathbb{R}^{N}} \int_{\mathbb{R}^{N}} d k_{0} d h_{0} \tilde{\rho}_{b}\left(k_{0}, h_{0}\right) e^{i R\left(k_{0}-h_{0}+\sum_{i=1}^{n} k_{i}+\sum_{j=1}^{m} h_{j}\right)} \\
& e^{-\frac{i \hbar}{2}\left\langle\left(-\frac{L^{N} C^{T} \gamma}{\hbar}-\frac{v_{\Omega_{B}, R}}{\hbar}-\frac{v_{C x}}{\hbar}+k_{0} G_{0}+\sum_{i=1}^{n} k_{i} G_{s_{i}}\right),\left(I_{N}-L_{B}\right)^{-1}\left(-\frac{L^{N} C^{T} \gamma}{\hbar}-\frac{v_{\Omega_{B}, R}}{\hbar}-\frac{v_{C x}}{\hbar}+k_{0} G_{0}+\sum_{i=1}^{n} k_{i} G_{s_{i}}\right)\right\rangle} \\
& e^{+\frac{i \hbar}{2}\left\langle\left(-\frac{L^{N} C^{T} \gamma^{\prime}}{\hbar}-\frac{v_{\Omega_{B}, R}}{\hbar}-\frac{v_{C y}}{\hbar}+h_{0} G_{0}-\sum_{s} j=1^{m} h_{j} G_{r_{j}}\right),\left(I_{N}-L_{B}\right)^{-1}\left(-\frac{L^{N} C^{T} \gamma^{\prime}}{\hbar}-\frac{v_{\Omega_{B}, R}}{\hbar}-\frac{v_{C y}}{\hbar}+h_{0} G_{0}-\sum_{j=1}^{m} h_{j} G_{r_{j}}\right)\right\rangle} \\
& \text { where } v_{B}(R)=\int_{\mathbb{R}^{N}} e^{i k R} d \mu_{v}(R), \rho_{B}(R, Q)=\int_{\mathbb{R}^{N}} \int_{\mathbb{R}^{N}} e^{i k_{0} R-i h_{0} Q} \tilde{\rho}_{B}\left(k_{0}, h_{0}\right) d k_{0} d h_{0}, \\
& v_{\Omega_{B}, R}, v_{C x}, G_{s} \in \mathcal{H}_{t}^{N}, s \in[0, t] \text {, are defined by } \\
& \left\langle v_{\Omega_{B}, R}, \Gamma\right\rangle=\int_{0}^{t} R \Omega_{B}^{2} \Gamma(s) d s \\
& \left\langle v_{C X}, \Gamma\right\rangle=\int_{0}^{t} x C \Gamma(s) d s, \\
& \left\langle G_{s}, \Gamma\right\rangle=\Gamma(s) .
\end{aligned}
$$

By Fubini theorem we have:

$$
\begin{aligned}
& F\left(\gamma, \gamma^{\prime}, x, y\right)=\left|\operatorname{det}\left(I_{N}-L_{B}\right)\right|^{-1} \lim _{\epsilon \rightarrow 0^{+}} \sum_{n=0}^{\infty} \sum_{m=0}^{\infty} \frac{1}{n !} \frac{1}{m !}\left(\frac{-i}{\hbar}\right)^{n}\left(\frac{i}{\hbar}\right)^{m} \\
& \int_{0}^{t} \cdots \int_{0}^{t} \int_{0}^{t} \ldots \int_{0}^{t} \prod_{i=1}^{n} d s_{i} \prod_{j=1}^{m} d r_{j} \int_{\mathbb{R}^{N}} \ldots \int_{\mathbb{R}^{N}} \int_{\mathbb{R}^{N}} \ldots \int_{\mathbb{R}^{N}} \prod_{i=1}^{n} d \mu_{v}\left(k_{i}\right) \prod_{j=1}^{m} d \mu_{v}\left(h_{j}\right) \\
& \int_{\mathbb{R}^{N}} \int_{\mathbb{R}^{N}} d k_{0} d h_{0} \rho_{b}\left(k_{0}, h_{0}\right) g_{1}\left(\gamma^{\prime}\right) \bar{g}_{1}(\gamma) g_{2}\left(\gamma^{\prime}, h_{0},-\mathbf{h}, \mathbf{r}, y\right) \bar{g}_{2}\left(\gamma, k_{0}, \mathbf{k}, \mathbf{s}, x\right) \\
& \int_{\mathbb{R}^{N}} d R e^{-\epsilon R^{2}} e^{-\frac{i}{\hbar} \int_{0}^{t}\left(\gamma(s)+x-\gamma^{\prime}(s)-y\right) C R d s} e^{i R\left(k_{0}-h_{0}+\sum_{i=1}^{n} k_{i}+\sum_{j=1}^{m} h_{j}\right)} \\
& e^{\left.-\frac{i}{\hbar} R \int_{0}^{t} \Omega_{B}^{2}\left(I-L_{B}\right)^{-1} L^{N} C^{T}\left(\gamma(s)-\gamma^{\prime}(s)\right) d s\right)} e^{i R \int_{0}^{t} \Omega_{B}^{2}\left(I-L_{B}\right)^{-1}\left(-\frac{v_{C}, x}{\hbar}+\frac{v_{C, y}}{\hbar}+\left(k_{0}-h_{0}\right) G_{0}+\sum_{i=1}^{n} k_{i} G_{s_{i}}+\sum_{j=1}^{m} h_{j} G_{r_{j}}\right)}
\end{aligned}
$$

where, for every paths $\gamma, \gamma^{\prime}, x \in \mathbb{R}^{n}, v_{0} \in \mathbb{R}$ and vectors $\mathbf{v}=\left(v_{1}, \ldots, v_{n}\right)$, $\mathbf{w}=\left(w_{1}, \ldots, w_{n}\right)$ we have defined the functions:

$$
g_{1}(\gamma):=e^{+\frac{i}{2 \hbar}\left\langle L^{N} C^{T} \gamma,\left(I_{N}-L_{B}\right)^{-1} L^{N} C^{T} \gamma\right\rangle}
$$


and

$$
\begin{aligned}
g_{2}\left(\gamma, v_{0}, \mathbf{v}, \mathbf{w}, x\right) & :=e^{+\frac{i \hbar}{2}\left\langle v_{0} G_{0}+\sum_{i=1}^{n} v_{i} G_{w_{i}}-\frac{v_{C, x}}{\hbar},\left(I_{N}-L_{B}\right)^{-1}\left(v_{0} G_{0}+\sum_{i=1}^{n} v_{i} G_{w_{i}}-\frac{v_{C, x}}{\hbar}\right)\right\rangle} \times \\
& \times e^{-i\left\langle L^{N} C^{t} \gamma,\left(I_{N}-L_{B}\right)^{-1}\left(v_{0} G_{0}+\sum_{i=1}^{n} v_{i} G_{w_{i}}-\frac{v_{C}, x}{\hbar}\right)\right\rangle}
\end{aligned}
$$

By integrating with respect to $R$ we have that (50) the latter is equal to:

$$
\begin{aligned}
& \left|\operatorname{det}\left(I_{N}-L_{B}\right)\right|^{-1} \lim _{\epsilon \rightarrow 0^{+}} \sum_{n=0}^{\infty} \sum_{m=0}^{\infty} \frac{1}{n !} \frac{1}{m !}\left(\frac{-i}{\hbar}\right)^{n}\left(\frac{i}{\hbar}\right)^{m} \\
& \int_{0}^{t} \ldots \int_{0}^{t} \int_{0}^{t} \ldots \int_{0}^{t} \prod_{i=1}^{n} d s_{i} \prod_{j=1}^{m} d r_{j} \int_{\mathbb{R}^{N}} \ldots \int_{\mathbb{R}^{N}} \int_{\mathbb{R}^{N}} \ldots \int_{\mathbb{R}^{N}} \prod_{i=1}^{n} d \mu_{v}\left(k_{i}\right) \prod_{j=1}^{m} d \mu_{v}\left(h_{j}\right) \\
& \int_{\mathbb{R}^{N}} \int_{\mathbb{R}^{N}}\left(\frac{\pi}{\epsilon}\right)^{N / 2}\left(e^{-\frac{\omega^{2}}{4 \epsilon}}\right) \tilde{\rho}_{b}\left(k_{0}, h_{0}\right) g_{1}\left(\gamma^{\prime}\right) \bar{g}_{1}(\gamma) g_{2}\left(\gamma^{\prime}, h_{0},-\mathbf{h}, \mathbf{r}, y\right) \bar{g}_{2}\left(\gamma, k_{0}, \mathbf{k}, \mathbf{s}, x\right) d k_{0} d h_{0}
\end{aligned}
$$

were:

$$
\begin{aligned}
\omega & :=\mid-\frac{1}{\hbar} \int_{0}^{t}\left(I-L_{B}\right)^{-1} C^{T}\left(\gamma(s)-\gamma^{\prime}(s)\right) d s-\frac{1}{\hbar}\left(\Omega_{B} \cos \Omega_{B} t\right)^{-1} \sin \left(\Omega_{B} t\right) C^{T}(x-y)+ \\
& +\left.\left(\cos \Omega_{B} t\right)^{-1}\left(k_{0}-h_{0}+\sum_{i=1}^{n} \cos \left(\Omega_{B} s_{i}\right) k_{i}+\sum_{j=1}^{m} \cos \left(\Omega_{B} r_{j}\right) h_{j}\right)\right|^{2}
\end{aligned}
$$

By introducing the new integration variables:

$$
k_{0}^{\prime}:=\frac{1}{\sqrt{\epsilon}}\left(k_{0}-h_{0}+a\right), \quad h_{0}^{\prime}:=h_{0}-\frac{1}{2} a
$$

with:

$$
\begin{aligned}
a:= & \sum_{i=1}^{n} \cos \left(\Omega_{B} s_{i}\right) k_{i}+\sum_{j=1}^{m} \cos \left(\Omega_{B} r_{j}\right) h_{j}-\cos \left(\Omega_{B} t\right) \frac{1}{\hbar} \int_{0}^{t}\left(I-L_{B}\right)^{-1} C^{T}(\gamma(s)+ \\
& \left.\left.\left.-\gamma^{\prime}(s)\right) d s\right)-\frac{1}{\hbar}\left(\Omega_{B}\right)^{-1} \sin \left(\Omega_{B} t\right) C^{T}(x-y)\right)
\end{aligned}
$$

where:

$$
\int_{0}^{t}\left(I-L_{B}\right)^{-1} C^{T}\left(\gamma(s)-\gamma^{\prime}(s)\right) d s=\cos ^{-1}\left(\Omega_{B} t\right) \int_{0}^{t} \cos \left(\Omega_{B} s\right) C^{T}\left(\gamma(s)-\gamma^{\prime}(s)\right) d s
$$


the integral in (53), with $k_{0}=\sqrt{\epsilon} k_{0}^{\prime}+h_{0}^{\prime}-\frac{a}{2}$ and $h_{0}=h_{0}^{\prime}+\frac{a}{2}$, can be written as:

$$
\begin{gathered}
\pi^{N / 2}\left|\operatorname{det}\left(I_{N}-L_{B}\right)\right|^{-1} \lim _{\epsilon \rightarrow 0^{+}} \sum_{n=0}^{\infty} \sum_{m=0}^{\infty} \frac{1}{n !} \frac{1}{m !}\left(\frac{-i}{\hbar}\right)^{n}\left(\frac{i}{\hbar}\right)^{m} \\
\int_{0}^{t} \ldots \int_{0}^{t} \int_{0}^{t} \ldots \int_{0}^{t} \prod_{i=1}^{n} d s_{i} \prod_{j=1}^{m} d r_{j} \int_{\mathbb{R}^{N}} \ldots \int_{\mathbb{R}^{N}} \int_{\mathbb{R}^{N}} \ldots \int_{\mathbb{R}^{N}} \prod_{i=1}^{n} d \mu_{v}\left(k_{i}\right) \prod_{j=1}^{m} d \mu_{v}\left(h_{j}\right) \\
\int_{\mathbb{R}^{N}} \int_{\mathbb{R}^{N}} d k_{0}^{\prime} d h_{0}^{\prime} \tilde{\rho}_{b}\left(\sqrt{\epsilon} k_{0}^{\prime}+h_{0}^{\prime}-\frac{1}{2} a, h_{0}^{\prime}+\frac{1}{2} a\right) g_{1}\left(\gamma^{\prime}\right) \bar{g}_{1}(\gamma) \\
g_{2}\left(\gamma^{\prime}, h_{0}^{\prime}+\frac{a}{2},-\mathbf{h}, \mathbf{r}, y\right) \bar{g}_{2}\left(\gamma, \sqrt{\epsilon} k_{0}^{\prime}+h_{0}^{\prime}-\frac{a}{2}, \mathbf{k}, \mathbf{s}, x\right) e^{-\frac{1}{4}\left|\left(\cos \Omega_{B} t\right)^{-1} k_{0}^{\prime}\right|^{2}}
\end{gathered}
$$

By letting $\epsilon \rightarrow 0$ and using dominated convergence, the integral reduces to the following form:

$$
\begin{aligned}
& F\left(\gamma, \gamma^{\prime}, x, y\right)=K(x, y, t) e^{-\frac{i}{2 \hbar}\left\langle\left(\gamma-\gamma^{\prime}\right), A\left(\gamma+\gamma^{\prime}\right)\right\rangle} e^{-\frac{i}{\hbar}\left\langle\gamma, C L^{N}\left(I_{N}-L_{B}\right)^{-1} v_{C, x}\right\rangle} \\
& e^{\frac{i}{\hbar}\left\langle\gamma^{\prime}, C L^{N}\left(I_{N}-L_{B}\right)^{-1} v_{C, y}\right\rangle} e^{\frac{i}{2 \hbar} C^{T}(x-y) \int_{0}^{t} \frac{\sin \left(\Omega_{B} t\right) \sin \left(\Omega_{B}(t-s)\right)}{\Omega_{B}^{2} \cos \left(\Omega_{B} t\right)} C^{T}\left(\gamma(s)+\gamma^{\prime}(s)\right) d s} \\
& \sum_{n=0}^{\infty} \sum_{m=0}^{\infty} \frac{1}{n !} \frac{1}{m !}\left(\frac{-i}{\hbar}\right)^{n}\left(\frac{i}{\hbar}\right)^{m} \int_{0}^{t} \ldots \int_{0}^{t} \int_{0}^{t} \ldots \int_{0}^{t} \prod_{i=1}^{n} d s_{i} \prod_{j=1}^{m} d r_{j} \int_{\mathbb{R}^{N}} \ldots \int_{\mathbb{R}^{N}} \\
& \int_{\mathbb{R}^{N}} \ldots \int_{\mathbb{R}^{N}} \prod_{i=1}^{n} d \mu_{v}\left(k_{i}\right) \prod_{j=1}^{m} d \mu_{v}\left(h_{j}\right) \int_{\mathbb{R}^{N}} d h_{0}^{\prime} \tilde{\rho}_{b}\left(h_{0}^{\prime}-\frac{1}{2} a, h_{0}^{\prime}+\frac{1}{2} a\right) \\
& e^{-\frac{i \hbar}{2} \sum_{i, j=1}^{n} k_{i} \frac{\sin \left(\Omega_{B}\left(t-s_{i} \vee s_{j}\right)\right) \cos \left(\Omega_{B}\left(s_{i} \wedge s_{j}\right)\right)}{\Omega_{B} \cos \left(\Omega_{B} t\right)} k_{j}} e^{\frac{i \hbar}{2} \sum_{i, j=1}^{m} h_{i} \frac{\sin \left(\Omega_{B}\left(t-r_{i} \vee r_{j}\right)\right) \cos \left(\Omega_{B}\left(r_{i} \wedge r_{j}\right)\right)}{\Omega_{B} \cos \left(\Omega_{B} t\right)} h_{j}} \\
& e^{i \sum_{i=1}^{n} k_{i} \frac{\cos \left(\Omega_{B} s_{i}\right)-\cos \left(\Omega_{B} t\right)}{\Omega_{B}^{2} \cos \left(\Omega_{B} t\right)} C^{T} x} e^{i \sum_{j=1}^{m} h_{j} \frac{\cos \left(\Omega_{B} r_{j}\right)-\cos \left(\Omega_{B} t\right)}{\Omega_{B}^{2} \cos \left(\Omega_{B} t\right)} C^{T} y} \\
& e^{i\left(h_{0}-\frac{a}{2}\right) \frac{1-\cos \left(\Omega_{B} t\right)}{\Omega_{B}^{2} \cos \left(\Omega_{B} t\right)} C^{T} x} e^{-i\left(h_{0}+\frac{a}{2}\right) \frac{1-\cos \left(\Omega_{B} t\right)}{\Omega_{B}^{2} \cos \left(\Omega_{B} t\right)} C^{T} y} \\
& e^{-\frac{i}{2} \int_{0}^{t} \frac{\sin \left(\Omega_{B}(t-s)\right)}{\Omega_{B} \cos \left(\Omega_{B} t\right)} C^{T}\left(\gamma(s)+\gamma^{\prime}(s)\right) d s\left(\sum_{i=1}^{n} \cos \left(\Omega_{B} s_{i}\right) k_{i}+\sum_{j=1}^{m} \cos \left(\Omega_{B} r_{j}\right) h_{j}\right)} \\
& e^{i \hbar h_{0}^{\prime} \frac{\sin \Omega_{B} t}{\Omega_{B} \cos \Omega_{B} t} a} e^{-i \hbar\left(h_{0}^{\prime}-a / 2\right) \sum_{i=1}^{n} \frac{\sin \left(\Omega_{B}\left(t-s_{i}\right)\right)}{\Omega_{B} \cos \left(\Omega_{B} t\right)} k_{i}} e^{-i \hbar\left(h_{0}^{\prime}+a / 2\right) \sum_{j=1}^{m} \frac{\sin \left(\Omega_{B}\left(t-r_{j}\right)\right)}{\Omega_{B} \cos \left(\Omega_{B} t\right)} h_{j}} \\
& e^{i\left\langle\gamma, \sum_{i=1}^{n} C L^{N}\left(I_{N}-L_{B}\right)^{-1} k_{i} G_{s_{i}}\right\rangle} e^{i\left\langle\gamma^{\prime}, \sum_{j=1}^{m} C L^{N}\left(I_{N}-L_{B}\right)^{-1} h_{j} G_{r_{j}}\right\rangle} e^{i\left\langle\gamma-\gamma^{\prime}, C L^{N}\left(I_{N}-L_{B}\right)^{-1} h_{0}^{\prime} G_{0}\right\rangle}
\end{aligned}
$$

where we have defined:

$$
K(x, y, t):=\pi^{N} 2^{N} e^{-\frac{i}{2 \hbar} C^{T}(x-y)\left(\frac{t}{\Omega_{B}^{2}}-\frac{\sin \left(\Omega_{B} t\right)}{\Omega_{B}^{3} \cos \left(\Omega_{B} t\right)}\right) C^{T}(x-y)}
$$


and

$$
\begin{aligned}
& e^{-\frac{i}{2 \hbar}\left\langle\left(\gamma-\gamma^{\prime}\right), A\left(\gamma+\gamma^{\prime}\right)\right\rangle}:=e^{-\frac{i}{2 \hbar}\left\langle C^{T}\left(\gamma-\gamma^{\prime}\right), L^{N}\left(I_{N}-L_{B}\right)^{-1} L^{N} C^{T}\left(\gamma+\gamma^{\prime}\right)\right\rangle} \times \\
& \times e^{+\frac{i}{2 \hbar} \cos \left(\Omega_{B} t\right)\left\langle C^{T}\left(\gamma(s)-\gamma^{\prime}(s)\right),\left(I_{N}-L_{B}\right)^{-1} v\right\rangle\left\langle L^{N}\left(I_{N}-L_{B}\right)^{-1} G_{0}, C^{T}\left(\gamma(s)+\gamma^{\prime}(s)\right)\right\rangle}= \\
& =e^{\frac{i}{2 \hbar} \int_{0}^{t} C^{T}\left(\gamma-\gamma^{\prime}\right)(s) \Omega^{-1} \int_{0}^{s} \sin \left(\Omega_{B}(s-r)\right) C^{T}\left(\gamma+\gamma^{\prime}\right)(r) d r d s}
\end{aligned}
$$

with $v(s)_{i}:=\frac{t^{2}-s^{2}}{2}, i=1 \ldots N, s \in[0, t]$.

As we have assumed that the determinant of the $d \times d$ left upper block of the $n \times n$ matrix $\cos (\Omega t)\left(\Omega^{2}\right.$ being the matrix $\left.(28)\right)$ is non vanishing, it is possible to prove (see appendix A) that the operator $I-L_{A}-A$ is invertible. As $F\left(\gamma, \gamma^{\prime}, x, y\right)$ is of the form $F\left(\gamma, \gamma^{\prime}\right)=$ $e^{-\frac{i}{2 \hbar}\left\langle\left(\gamma-\gamma^{\prime}\right), A\left(\gamma+\gamma^{\prime}\right)\right\rangle} f\left(\gamma, \gamma^{\prime}\right)$, with $f \in \mathcal{F}\left(\mathcal{H}_{t}^{d} \oplus \mathcal{H}_{t}^{d}\right)$, we can conclude that the influence functional is a Fresnel integrable function.

Second step Let us prove that the reduced density operator $\rho_{R}(t, x, y)$ is given by the infinite dimensional oscillatory integral (48).

Let $\rho(t, x, y, R, Q)$ be the (smooth) kernel of the density operator of the compound system evaluated at time $t$. Then the integral giving the kernel of reduced density operator $\rho_{R}(t, x, y):=\int \rho(t, x, y, R, R) d R$ is absolutely convergent and by Lebesgue's dominated convergence theorem we have $\rho_{R}(t, x, y)=\lim _{\epsilon \rightarrow 0} \int \rho(t, x, y, R, R) e^{-\epsilon R^{2}} d R$.

On the other hand the influence functional can be written as $F\left(\gamma, \gamma^{\prime}\right)=$ $e^{-\frac{i}{2 \hbar}\left\langle\left(\gamma-\gamma^{\prime}\right), A\left(\gamma+\gamma^{\prime}\right)\right\rangle} f\left(\gamma, \gamma^{\prime}\right)$, with $f: \mathcal{H}_{t}^{d} \oplus \mathcal{H}_{t}^{d} \rightarrow \mathbb{C}$ defined as follows:

$$
f=\lim _{\epsilon \rightarrow 0} f_{\epsilon}
$$

and where:

$$
\begin{gathered}
f_{\epsilon}\left(\gamma, \gamma^{\prime}\right):=\pi^{N / 2}\left|\operatorname{det}\left(I_{N}-L_{B}\right)\right|^{-1} \lim _{\epsilon \rightarrow 0^{+}} \sum_{n=0}^{\infty} \sum_{m=0}^{\infty} \frac{1}{n !} \frac{1}{m !}\left(\frac{-i}{\hbar}\right)^{n}\left(\frac{i}{\hbar}\right)^{m} \\
\int_{0}^{t} \ldots \int_{0}^{t} \int_{0}^{t} \ldots \int_{0}^{t} \prod_{i=1}^{n} d s_{i} \prod_{j=1}^{m} d r_{j} \int_{\mathbb{R}^{N}} \ldots \int_{\mathbb{R}^{N}} \int_{\mathbb{R}^{N}} \ldots \int_{\mathbb{R}^{N}} \prod_{i=1}^{n} d \mu_{v}\left(k_{i}\right) \prod_{j=1}^{m} d \mu_{v}\left(h_{j}\right) \\
\int_{\mathbb{R}^{N}} \int_{\mathbb{R}^{N}} d k_{0}^{\prime} d h_{0}^{\prime} \tilde{\rho}_{b}\left(\sqrt{\epsilon} k_{0}^{\prime}+h_{0}^{\prime}-\frac{1}{2} a, h_{0}^{\prime}+\frac{1}{2} a\right) \\
g_{2}\left(\gamma^{\prime}, h_{0}^{\prime}+\frac{a}{2},-\mathbf{h}, \mathbf{r}, y\right) \bar{g}_{2}\left(\gamma, \sqrt{\epsilon} k_{0}^{\prime}+h_{0}^{\prime}-\frac{a}{2}, \mathbf{k}, \mathbf{s}, x\right) e^{-\frac{1}{4}\left|\left(\cos \Omega_{B} t\right)^{-1} k_{0}^{\prime}\right|^{2}}
\end{gathered}
$$

with $\left.a^{\prime}:=a+\cos \left(\Omega_{B} t\right) \frac{1}{\hbar} \int_{0}^{t}\left(I-L_{B}\right)^{-1} C^{T}\left(\gamma(s)-\gamma^{\prime}(s)\right) d s\right)$ and the limit (57) is meant in the $\mathcal{F}\left(\mathcal{H}_{t}^{d} \oplus \mathcal{H}_{t}^{d}\right)$ sense.

By the continuity of the infinite dimensional oscillatory integral as a functional on $\mathcal{F}\left(\mathcal{H}_{t}^{d} \oplus \mathcal{H}_{t}^{d}\right)$ (see corollary 2) we have that the r.h.s of 
equation (48) is equal to:

$$
\begin{array}{r}
e^{-\frac{i t}{2 \hbar} x \Omega_{A}^{2} x} e^{\frac{i t}{2 \hbar} y \Omega_{A}^{2} y} \lim _{\epsilon \rightarrow 0} \widetilde{\int_{\mathcal{H}_{t}^{d}}} \widetilde{\int_{\mathcal{H}_{t}^{d}}} e^{\frac{i}{2 \hbar}\left\langle\gamma,\left(I_{d}-L_{A}\right) \gamma\right\rangle} e^{-\frac{i}{2 \hbar}\left\langle\gamma^{\prime},\left(I_{d}-L_{A}\right) \gamma^{\prime}\right\rangle} \\
e^{-\frac{i}{\hbar} \int_{0}^{t} x \Omega_{A}^{2} \gamma(s) d s} e^{\frac{i}{\hbar} \int_{0}^{t} y \Omega_{A}^{2} \gamma^{\prime}(s) d s} e^{-\frac{i}{\hbar} \int_{0}^{t} v_{A}(\gamma(s)+x) d s+\frac{i}{\hbar} \int_{0}^{t} v_{A}\left(\gamma^{\prime}(s)+y\right) d s} \\
e^{-\frac{i}{2 \hbar}\left\langle\left(\gamma-\gamma^{\prime}\right), A\left(\gamma+\gamma^{\prime}\right)\right\rangle} f_{\epsilon}\left(\gamma, \gamma^{\prime}\right) \rho_{0}^{A}\left(\gamma(0)+x, \gamma^{\prime}(0)+y\right) d \gamma d \gamma^{\prime}
\end{array}
$$

On the other hand the latter is equal to:

$$
\begin{gathered}
e^{-\frac{i t}{2 \hbar} x \Omega_{A}^{2} x} e^{\frac{i t}{2 \hbar} y \Omega_{A}^{2} y} \lim _{\epsilon \rightarrow 0} \widetilde{\int_{\mathcal{H}_{t}^{d}}} \widetilde{\int_{\mathcal{H}_{t}^{d}}} e^{\frac{i}{2 \hbar}\left\langle\gamma,\left(I_{d}-L_{A}\right) \gamma\right\rangle} e^{-\frac{i}{2 \hbar}\left\langle\gamma^{\prime},\left(I_{d}-L_{A}\right) \gamma^{\prime}\right\rangle} \\
e^{-\frac{i}{\hbar} \int_{0}^{t} x \Omega_{A}^{2} \gamma(s) d s} e^{\frac{i}{\hbar} \int_{0}^{t} y \Omega_{A}^{2} \gamma^{\prime}(s) d s} e^{-\frac{i}{\hbar} \int_{0}^{t} v_{A}(\gamma(s)+x) d s+\frac{i}{\hbar} \int_{0}^{t} v_{A}\left(\gamma^{\prime}(s)+y\right) d s} \\
\widetilde{\int_{\mathcal{H}_{t}^{N}}} \widetilde{\int_{\mathcal{H}_{t}^{N}}} e^{\frac{i}{2 \hbar}\left\langle\Gamma,\left(I_{N}-L_{B}\right) \Gamma\right\rangle} e^{-\frac{i}{2 \hbar}\left\langle\Gamma^{\prime},\left(I_{N}-L_{B}\right) \Gamma^{\prime}\right\rangle} e^{-\frac{i}{\hbar}\left\langle\Gamma, L^{N} C^{T} \gamma\right\rangle} e^{\frac{i}{\hbar}\left\langle\Gamma^{\prime}, L^{N} C^{T} \gamma^{\prime}\right\rangle} \\
e^{-\frac{i}{\hbar} \int_{0}^{t} v_{B}(\Gamma(s)+R) d s+\frac{i}{\hbar} \int_{0}^{t} v_{B}\left(\Gamma^{\prime}(s)+R\right) d s} e_{0}^{-\frac{i t}{\hbar} x C R} e^{+\frac{i t}{\hbar} y C R} e^{-\frac{i}{\hbar} \int_{0}^{t}\left(\gamma(s)-\gamma^{\prime}(s)\right) C R d s} \\
e^{-\frac{i}{\hbar} \int_{0}^{t} R \Omega_{B}^{2}\left(\Gamma(s)-\Gamma^{\prime}(s)\right) d s} e^{-\frac{i}{\hbar} \int_{0}^{t}\left(x C \Gamma(s)+y C \Gamma^{\prime}(s)\right) d s}
\end{gathered}
$$

By Fubini theorem (see theorem 3) and by the infinite dimensional oscillatory integral representation or the kernel of the density operator it is equal to $\int d R e^{-\epsilon R^{2}} \rho(t, x, y, R, R)$. By letting $\epsilon \rightarrow 0$ the conclusion follows.

Remark 2. It is typical of the difficulties in handling rigorously Feynman path integrals (as infinite dimensional oscillatory integrals) that the passages to the limit cause mathematical problems, because of the lack of the dominated convergence and limited avalability of Fubini-type theorems. Our $\epsilon$-cut-off trick was instrumental to perform such type of computation.

\section{Application to the Caldeira-Leggett model}

Let us compute now the influence functional $F\left(\gamma, \gamma^{\prime}, x, y\right)$ in the case $v_{B} \equiv 0, \rho_{0}^{B}(R, Q):=\prod_{j=1}^{N} \rho_{B}^{(j)}\left(R_{j}, Q_{j}, 0\right)$, where:

$$
\rho_{B}^{(j)}\left(R_{j}, Q_{j}, 0\right):=\sqrt{\left.\frac{m \omega_{j}}{\pi \hbar \operatorname{coth}\left(\hbar \omega_{j} / 2 k T\right.}\right)} e^{-\left(\frac{m \omega_{j}}{2 \hbar \sinh \left(\hbar \omega_{j} / k T\right)}\left(\left(R_{j}^{2}+Q_{j}^{2}\right) \cosh \frac{\hbar \omega_{j}}{k T}-2 R_{j} Q_{j}\right)\right)}
$$


$\omega_{j}, j=1 \ldots n$ being the eigenvalues of the matrix $\Omega_{B}$. By notation simplicity we put $m=1$, the general case can be handled by replacing $\Omega_{A}, \Omega_{B}, C, v_{A}, v_{B}, \rho_{0}^{A}, \rho_{0}^{B}$ with $\Omega_{A}^{\prime}, \Omega_{B}^{\prime}, C^{\prime}, v_{A}^{\prime}, v_{B}^{\prime}, \rho_{0, A}^{\prime}, \rho_{0, B}^{\prime}$.

By inserting this into the general formula (55) the influence functional becomes:

$$
\begin{gathered}
F\left(\gamma, \gamma^{\prime}, x, y\right)=K(x, y, t) e^{-\frac{i}{2 \hbar}\left\langle\left(\gamma-\gamma^{\prime}\right), A\left(\gamma+\gamma^{\prime}\right)\right\rangle} e^{-\frac{i}{\hbar}\left\langle\gamma, C L^{N}\left(I_{N}-L_{B}\right)^{-1} v_{C, x}\right\rangle} \\
e^{\frac{i}{\hbar}\left\langle\gamma^{\prime}, C L^{N}\left(I_{N}-L_{B}\right)^{-1} v_{C, y}\right\rangle} e^{\frac{i}{2 \hbar} C^{T}(x-y) \int_{0}^{t} \frac{\sin \left(\Omega_{B} t\right) \sin \left(\Omega_{B}(t-s)\right)}{\Omega_{B}^{2} \cos \left(\Omega_{B} t\right)} C^{T}\left(\gamma(s)+\gamma^{\prime}(s)\right) d s} \\
\int_{\mathbb{R}^{N}} d h_{0}^{\prime} \tilde{\rho}_{b}\left(h_{0}^{\prime}-\frac{1}{2} a, h_{0}^{\prime}+\frac{1}{2} a\right) e^{i\left(h_{0}-\frac{a}{2}\right) \frac{1-\cos \left(\Omega_{B} t\right)}{\Omega_{B}^{2} \cos \left(\Omega_{B} t\right)} C^{T} x} e^{-i\left(h_{0}+\frac{a}{2}\right) \frac{1-\cos \left(\Omega_{B} t\right)}{\Omega_{B}^{2} \cos \left(\Omega_{B} t\right)} C^{T} y} \\
e^{i \hbar h_{0}^{\prime} \frac{\sin \Omega_{B} t}{\Omega_{B} \cos \Omega_{B} t} a} e^{i\left\langle\gamma-\gamma^{\prime}, C L^{N}\left(I_{N}-L_{B}\right)^{-1} h_{0}^{\prime} G_{0}\right\rangle}
\end{gathered}
$$

where

$$
\begin{gathered}
K(x, y, t):=\pi^{N} 2^{N} e^{\frac{i}{2 \hbar} C^{T}(x-y)\left(\frac{t}{\Omega_{B}^{2}}-\frac{\sin \left(\Omega_{B} t\right)}{\Omega_{B}^{3}}\right) C^{T}(x+y)} \\
e^{-\frac{i}{2 \hbar}\left\langle\left(\gamma-\gamma^{\prime}\right), A\left(\gamma+\gamma^{\prime}\right)\right\rangle}:=e^{-\frac{i}{2 \hbar}\left\langle C^{T}\left(\gamma-\gamma^{\prime}\right), L^{N}\left(I_{N}-L_{B}\right)^{-1} L^{N} C^{T}\left(\gamma+\gamma^{\prime}\right)\right\rangle} \\
e^{+\frac{i}{2 \hbar} \cos \left(\Omega_{B} t\right)\left\langle C^{T}\left(\gamma(s)-\gamma^{\prime}(s)\right),\left(I_{N}-L_{B}\right)^{-1} v\right\rangle\left\langle L^{N}\left(I_{N}-L_{B}\right)^{-1} G_{0}, C^{T}\left(\gamma(s)+\gamma^{\prime}(s)\right)\right\rangle} \\
=e^{\frac{i}{2 \hbar} \int_{0}^{t} C^{T}\left(\gamma-\gamma^{\prime}\right)(s) \Omega^{-1} \int_{0}^{s} \sin \left(\Omega_{B}(s-r)\right) C^{T}\left(\gamma+\gamma^{\prime}\right)(r) d r d s} \quad(60) \\
\left.\left.a=-\cos \left(\Omega_{B} t\right) \frac{1}{\hbar} \int_{0}^{t}\left(I-L_{B}\right)^{-1} C^{T}\left(\gamma(s)-\gamma^{\prime}(s)\right) d s\right)-\frac{1}{\hbar}\left(\Omega_{B}\right)^{-1} \sin \left(\Omega_{B} t\right) C^{T}(x-y)\right)
\end{gathered}
$$

By direct computation, we obtain:

$$
\begin{aligned}
& F\left(\gamma, \gamma^{\prime}, x, y\right)=e^{\frac{i}{2 \hbar} \int_{0}^{t} C^{T}\left(\gamma(s)+x-\gamma^{\prime}(s)-y\right) \Omega_{B}^{-1} \int_{0}^{s} \sin \left(\Omega_{B}(s-r)\right) C^{T}\left(\gamma(r)+x+\gamma^{\prime}(r)+y\right) d r d s} \\
& e^{-\frac{1}{2 \hbar} \int_{0}^{t} C^{T}\left(\gamma(s)+x-\gamma^{\prime}(s)-y\right) \Omega_{B}^{-1} \operatorname{coth}\left(\frac{\hbar \Omega_{B}}{2 k T}\right) \int_{0}^{s} \cos \left(\Omega_{B}(s-r)\right) C^{T}\left(\gamma(r)+x-\gamma^{\prime}(r)-y\right) d r d s}
\end{aligned}
$$

which yelds the result heuristically derived in [FV63].

Appendix A. Kernel of the operator $I-L_{A}-A$

A vector $\gamma \in \mathcal{H}_{t}^{d}$ belongs to the kernel of the operator $I-L_{A}-A$, if it satisfies the following equation:

$$
\begin{aligned}
\gamma(s) & +\int_{s}^{t} d s^{\prime} \int_{0}^{s^{\prime}} d s^{\prime \prime} \int_{0}^{s^{\prime \prime}} C \Omega_{B}^{-1} \sin \left(\Omega_{B}\left(s^{\prime \prime}-r\right)\right) C^{T} \gamma(r) d r+ \\
& -\int_{s}^{t} d s^{\prime} \int_{0}^{s^{\prime}} \Omega_{A}^{2} \gamma\left(s^{\prime \prime}\right) d s^{\prime \prime}=0 \quad s \in[0, t]
\end{aligned}
$$


with $\gamma(t)=0$. Equation (62) is equivalent to:

$$
\ddot{\gamma}(s)+\Omega_{A}^{2} \gamma(s)-\int_{0}^{s} C \Omega_{B}^{-1} \sin \left(\Omega_{B}(s-r)\right) C^{T} \gamma(r) d r=0
$$

with the conditions: $\gamma(t)=0, \dot{\gamma}(0)=0$.

By differentiating equation (62), it is easy to see that its solution, if it exists, is a $C^{\infty}$ function and its odd derivatives, evaluated for $s=0$, vanish, while the even derivatives satisfy the following relation

$$
\gamma^{2(N+2)}(0)+\Omega_{A}^{2} \gamma^{2(N+1)}(0)-\sum_{k=0}^{N}(-1)^{k} C \Omega_{B}^{2 k} C^{T} \gamma^{2(N-k)}(0)=0
$$

By induction it is possible to prove that $\gamma^{2 N}(0)=(-1)^{N}\left[\Omega^{2 N}\right]_{d \times d} \gamma(0)$, where $\left[\Omega^{2 N}\right]_{d \times d}$ denotes the $d \times d$ left upper block of the $N$-th power of the $n \times n$ matrix $\Omega^{2}$ (where $\Omega^{2}$ is given by equation (28)). One can conclude that the solution of equation (62) is of the form $\gamma(s)=$ $[\cos (\Omega s)]_{d \times d} \gamma(0)$. By imposing the condition $\gamma(t)=0$, one concludes that if $\operatorname{det}\left([\cos (\Omega s)]_{d \times d}\right) \neq 0$ then equation (62) cannot admit nontrivial solutions and the operator $I-L_{A}-A$ is invertible.

\section{Aknowledgements}

We would like to thank the hospitality of the mathematics departments in Bonn and Trento. L. Cattaneo gratefully acknowledges the financial support by the Lise Meitner Fellowship of the Land NordrheinWestfalen. S. Mazzucchi gratefully acknowledges the financial support by P.A.T. (Trento) and MIUR (Italy); this research is part of the I.D.A. project of INdAM.

\section{REFERENCES}

[AB93] S. Albeverio and Z. Brzeźniak. Finite-dimensional approximation approach to oscillatory integrals and stationary phase in infinite dimensions. J. Funct. Anal, 113(1):177-244, 1993.

[ABHK82] S. Albeverio, Ph. Blanchard, and R. Høegh-Krohn. Feynman path integrals and the trace formula for the Schrödinger operators. Comm. Math. Phys., 83(1):4976, 1982.

[AGM03] S. Albeverio, G. Guatteri, and S. Mazzucchi. Representation of the belavkin equation via Feynman path integrals. Probab. Theory Relat. Fields, 125(3):365-380, 2003.

[AGM04] S. Albeverio, G. Guatteri, and S. Mazzucchi. Representation of the belavkin equation via phase space Feynman path integrals. Infin. Dimens. Anal. Quantum Probab. Relat. Top., 7(4):507-526, 2004.

[AHK76] S. Albeverio and R. Høegh-Krohn. Mathematical theory of Feynman path integrals, volume 523. Springer-Verlag, Berlin, 1976. 2nd Edition (with S. Mazzucchi), Springer-Verlag, Berlin 2006. 
[AHK77] S. Albeverio and R. Høegh-Krohn. Oscillatory integrals and the method of stationary phase in infinitely many dimensions, with applications to the classical limit of quantum mechanics. Invent. Math., 40(1):59-106, 1977.

[AM] S. Albeverio and S. Mazzucchi. Feynman path integrals for timedependent potentials. In 7th International Meeting on Stochastic Partial Differential Equations and Applications edited by G. Da Prato and L. Tubaro.

[AM04a] S. Albeverio and S. Mazzucchi. Generalized infinite-dimensional fresnel integrals. C. R. Acad. Sci. Paris, 338(3):225-259, 2004.

[AM04b] S. Albeverio and S. Mazzucchi. Some new developments in the theory of path integrals, with applications to quantum theory. J. Stat. Phys., 115(112):191-215, 2004.

[AM05a] S. Albeverio and S. Mazzucchi. Feynman path integrals for polynomially growing potentials. J. Funct. Anal., 221(1):83-121, 2005.

[AM05b] S. Albeverio and S. Mazzucchi. Generalized fresnel integrals. Bull. Sci. Math., 129(1):1-23, 2005.

[BDE95] A. Barenco, D. Deutsch, and A. Ekert. Universality in quantum computation. Proc. R. Soc. Lond. A, 449:669-677, 1995.

[BDK95] A. Bulgac, Giu Do Dang, and D. Kusnezov. Random matrix approach to quantum dissipation. Ann. Phys., 242(1):3468-3478, 1995.

[BDK96] A. Bulgac, Giu Do Dang, and D. Kusnezov. Random matrix approach to quantum dissipation. Phys. Rev. E, 54(4):3468-3478, 1996.

[BDK97] A. Bulgac, Giu Do Dang, and D. Kusnezov. Turbulent-like diffusion in complex quantum systems. Physics Letter A, 234(2):103-107, 1997.

[BDK98] A. Bulgac, Giu Do Dang, and D. Kusnezov. Dynamics of a simple quantum system in a complex environment. Phys. Rev. E, 58(1):196-214, 1998.

[BG03] S. Banerjee and R. Ghosh. General quantum brownian motion with initially correlated and nonlinearly coupled environment. Phys. Rev. E, 67(056120), 2003.

[BP02] H.P. Breuer and F. Petruccione. The Theory of Open Quantum Systems. Oxford University Press, 2002.

[Bru93] Brun. Quasiclassical equations of motion for nonlinear brownian systems. Phys. Rev. D, 47:33833393, 1993.

[BSZ92] L. Bönig, K. Schönhammer, and W. Zwerger. Influence-functional theory for a heavy particle in a Fermi gas. Phys. Rev. B, 46(2):855-860, 1992.

[Cal83] A. O. Caldeira. PhD thesis, University of Sussex, 1983. unpublished.

[CEFM00] F. Castella, L. Erdös, F. Frommlet, and P.A. Markowich. FokkerPlanck equations as scaling limits of reversible quantum systems. Journal of Statistical Physics, 100(3-4):543-601, 2000.

[CH87] A.O. Caldeira and P. Hedegärd. Quantum dynamics of a particle in a fermionic environment. Physica Scripta, 35:609-622, 1987.

[Che87] Y.C. Chen. A new method for quantum processes in fermionic heat baths. J. Stat. Phys., 49(3-4):811 - 826, 1987. 
30 S. ALBEVERIO ${ }^{1), 2), 3}$, L. CATTANEO ${ }^{1)}$, L. DI PERSIO ${ }^{2)}$, S. MAZZUCCHI ${ }^{2,4)}$

[CL81] A. O. Caldeira and A.J. Leggett. Influence of dissipation on quantum tunneling in macroscopic systems. Phys. Rev. Letters, 46(4):211-214, 1981.

[CL83a] A. O. Caldeira and A.J. Leggett. Path integral approach to quantum brownian motion. Physica A, 121:587-616, 1983.

[CL83b] A. O. Caldeira and A.J. Leggett. Quantum tunneling in a dissipative system. Ann. Phys., 149:374-456, 1983.

[CL85] A. O. Caldeira and A.J. Leggett. Influence of damping on quantum interference: An exactly soluble model. Phys. Rev. A, 31(2):1059-1062, 1985.

[CLL89] Y.-C. Chen, J. L. Lebowitz, and C. Liverani. Dissipative quantum dynamics in a boson bath. Phys. Rev. B, 40(7):46644682, 1989.

[Dav73] E. B. Davies. The harmonic oscillator in a heat bath. Communications in Mathematical Physics, 33(3):171-186, 1973.

[Deu89] D. Deutsch. Quantum computational networks. Proc. R. Soc. Lond. A, 425:73-90, 1989.

[Dir33] P.A.M. Dirac. Physikalische Zeitschrift der Sowjetunion. Int. J. Mod. Phy. B, Band 3(Heft 1), 1933. Reprinted in Selected Papers on Quantum Electrodynamics, Ed. J.Schwinger, Dover, New York, (1958) P312.

[Dir47] P.A.M. Dirac. The principles of Quantum Mechanics. Oxfors University Press, Oxford, 1947.

[DS98] M. Dubé and P.C.E. Stamp. Dynamics of a pair of interacting spins coupled to an environmental sea. Int. J. Mod. Phy. B, 12(11):11911245, 1998.

[ET84] D. Elworthy and A. Truman. Feynman maps, Cameron-Martin formulae and anharmonic oscillators. Ann. Inst. H. Poincaré Phys. Théor., 41(2):115-142, 1984.

[Exn85] P. Exner. Open Quantum Systems and Feynman Integrals. Oxford University Press, 1985. Oxford Mathematical Monographs.

[Fey42] R.P. Feynman. The principle of least square action in quantum mechanics. PhD thesis, Princeton University, Princeton, NJ, 1942.

[Fey48] R.P. Feynman. Space-time approach to non-relativistic quantum mechanics. Rev. Mod. Phys., 20:367-387, 1948.

[FH65] R.P. Feynman and A.R. Hibbs. Quantum mechanics and path integrals. Macgraw Hill, 1965.

[FV63] R.P. Feynman and F.L. Vernon. The theory of a general quantum system interacting with a linear dissipative system. Ann. Phys., 24:118$173,1963$.

$\left[\mathrm{GJZ}^{+} 03\right]$ D. Giulini, E. Joos, H.D. Zeh, C. Kiefer, Kupsch, and I.-O. Stamatescu. Decoherence and the Appearance of the Classical World in Quantum Theory (2nd edition). Springer-Verlag, Berlin, 2003.

[GSI88] H. Grabert, P. Schramm, and G.L. Ingold. Quantum brownian motion: The functional integral approach. Phys. Rep., 168:115-207, 1988.

[Gui84] F. Guinea. Friction and particle-hole pairs. Phys. Rev. Lett., 53:12681271, 1984.

[GZ04] C.W. Gardiner and P. Zoller. Quantum Noise. Springer-Berlin: Springer Series in Synergetics, 2004. 
[HA85] V. Hakim and V. Ambegaokar. Quantum theory of a free particle interacting with a linearly dissipative environment. Phys. Rev. A, 32(1):423434, 1985.

[HPZ92] B.L. Hu, J.P. Paz, and Y. Zhang. Quantum brownian motion in a general environment: Exact master equation with nonlocal dissipation and colored noise. Phys. Rev. D, 45(8):2843-2861, 1992.

[HPZ93] B.L. Hu, J.P. Paz, and Y. Zhang. Quantum brownian motion in a general environment. II: Nonlinear coupling and perturbative approach. Phys. Rev. D, 47(4):1576-1594, 1993.

[JL02] G.W .Johnson and M.L. Lapidus. The Feynman Integral and Feynman's Operational Calculus. Reidel, Dordrecht, 2002.

[Kle04] H. Kleinert. Path Integrals in Quantum Mechanics, Statistics, Polymer Physics, and Financial Markets (4th edition). World Scientific, Singapore, 2004.

$\left[\mathrm{LCD}^{+} 87\right]$ A.J. Leggett, S. Chakravarty, A.T. Dorsey, M.P.A. Fisher, A. Garg, and W. Zwerger. Dynamics of the dissipative two-state system. Rev. Modern Phys., 59(1):1-85, 1987.

[Leg84] A.J. Leggett. Quantum tunneling in presence of an arbitrary linear dissipation mechanism. Phys. Rev. B, 30(3):1208-1218, 1984.

[Lin76] G. Lindblad. On the generators of quantum dynamical semigroups. Comm. Math. Phys., 48(2):119-130, 1976.

[PZ99] J.P Paz and W.H. Zurek. Les Houches Lectures Session LII. in Coherent Matter Waves. North Holland, Amsterdam, 1999.

[SC87] C. Morais Smith and A. O. Caldeira. Generalized Feynman-Vernon approach to dissipative quantum systems. Phys. Rev. A, 36(7):3509$3511,1987$.

[SC90] C. Morais Smith and A. O. Caldeira. Application of the generalized Feynman-Vernon approach to a simple system: The damped harmonic oscillator. Phys. Rev. A, 41(6):3103-3115, 1990.

[Sch82] A. Schmid. On a quasiclassical Langevin equation. J. Low Temp. Phys., 49(5-6):609-626, 1982.

[SH04] K. Shiokawa and B. L. Hu. Qubit decoherence and non-markovian dynamics at low temperatures via an effective spin-boson model. Phys. Rev A., 70(062106), 2004.

[Str97] W.T. Strunz. Path integral, semiclassical and stochastic propagators for markovian open quantum system. J. Phys. A: Math. Gen., 30:40534064, 1997.

[Unr95] W.G. Unruh. Maintaining coherence in quantum computers. Phys. Rev. A, 51(2):992997, 1995.

[UZ89] W.G. Unruh and W. H. Zurek. Reduction of a wave packet in quantum brownian motion. Phys. Rev. D, 40(4):1071-1094, 1989.

[Wei99] U. Weiss. Quantum dissipative systems(2nd edition). World Scientific, Singapore, 1999. Series in Modern Condensed Matter Physics - Vol. 10.

[Zur82] W. H. Zurek. Environment-induced superselection rules. Phys. Rev. D, 26(8):1862-1880, 1982.

[Zwe87] W. Zwerger. Quantum effects in the current-voltage characteristic of a small Josephson junction. Phys. Rev. B, 35(10):4737-4753, 1987. 
32 S. ALBEVERIO ${ }^{1), 2), 3}$, L. CATTANEO ${ }^{1)}$, L. DI PERSIO ${ }^{2)}$, S. MAZZUCCHI ${ }^{2,4)}$

1) Institut für Angewandte Mathematik, Wegelerstr. 6, 53115 Bonn (D)

2) Dip. Matematica, Università di Trento, 38050 Povo (I)

3) BiBoS; IZKS; SFB611; CERFim (Locarno); Acc. Arch. (MendriSIO)

4) INdAM, Trento (I) 